Umberto Tecchiati

\title{
NUOVE RICERCHE SUL POPOLAMENTO PREISTORICO E PROTOSTORICO DI VAL BADIA E VAL GARDENA
}

\section{Premessa}

Il presente contributo illustra l'attività di ricognizione di superficie effettuata dal Dipartimento di Storia della Civiltà Europea-Università degli Studi di Trento, nel corso del mese di settembre 1992 in aree campione della Val Badia e della Val Gardena".

Tale ricerca di superficie si proponeva di ampliare le conoscenze disponibili sul popolamento preistorico di questo areale geografico, anche e soprattutto in rapporto ai dati scaturiti dall'esplorazione archeologica dell'abitato di Sotćiastel in alta Val Badia ${ }^{2}$.

Come abbiamo infatti avuto modo di osservare in altra sede (BAGOLINI \& TECCHIATI, 1993b), lo scavo nell'abitato di Sotćiastel ha aperto, com'era prevedibile in un'area di recente interesse da parte dell'archeologia, una serie di interrogativi ai quali non era e non è agevole rispondere attraverso i soli dati di scavo, ma la cui soluzione importa la comprensione globale del "sistema" territoriale di riferimento. In quest'ambito si inserisce una serie di osservazioni fatte, fin dalla seconda campagna di scavo a Sotćiastel (TECCHIATI, 1990), a livello della viabilità e della proprietà fondiaria tradizionali.

La prima parte della ricognizione di superficie ha interessato la Val di Marebbe e in particolar modo la porzione settentrionale di essa che dà sulla conca di San Lorenzo in Val Pusteria. La scelta di questo campione territoriale si è basata sulla necessità di contestualizzare il sito di Ćiatlins-Gschlier (nei pressi di Peraforada/Palfrad) che la letteratura ( $v$. infra) definiva come abitato antico,

1) L'attività si inscrive nel programma di ricerche sul territorio promossa dalla Cattedra di Paletnologia della Facoltà di Lettere (Prof. B. Bagolini) in collaborazione con la Soprintendenza Provinciale ai Beni Culturali di Bolzano e I' Istitut Ladin "Micurá de Rü". Nella stessa estate ha avuto luogo anche un "survey" in Val Senales e zone limitrofe, finalizzato alla contestualizzazione ambientale della scoperta dell' "Uomo del Similaun": per la collaborazione offerta anche in tale occasione dall' Istitut Ladin "Micurá de Rü" desideriamo esprimere in questa sede la nostra sincera riconoscenza.
2) Altre aree dolomitiche sono state sottoposte, nel frattempo, ad un severo esame territoriale. L' attività svolta in questo settore dalla Cooperativa Scavi e Restauri di Bolzano, su incarico delI' Istitut Ladin "Majon di Fascegn" di Vigo di Fassa, per quanto attiene alla Val di Fassa, e dell' Istitut Ladin "Micurá de Rü" per quanto attiene alla Val Gardena e alla Val Badia (per i quali cfr. BOMBONATO, 1993; ZAGO, 1993), rimane un sicuro punto di riferimento soprattutto per quanto riguarda la metodologia di approccio alla realtà territoriale. 
e al quale abbiamo dedicato due sondaggi esplorativi, numerose carotazioni e prospezioni a mezzo metal-detector ${ }^{3}$.

La ricerca è continuata, nella seconda parte del programma di attività 1992 , in Val Gardena. ${ }^{4)}$ Va sottolineato che la Val Gardena, rispetto alla Val Badia, presenta una situazione archeologicamente molto più ricca, riguardante in pratica tutte le epoche del passato soggette ad indagine archeologica (PRINOTH, 1988).

L'esistenza di un "tessuto connettivo" di siti, reperti sporadici, segnalazioni, oltre a quella di un tracciato viario presumibilmente assai antico, il "Troi Paian", conferiscono alla ricerca da campo premesse, almeno potenzialmente, assai più solide e concrete.

\section{Ricerche di superficie e sondaggi in Val Badia - Val di Marebbe.}

Il campione territoriale indagato durante le attività '92 si situa alla destra idrografica del torrente Gadera, tra la località in cui sorge il Castello di Michelsburg, ai limiti meridionali della conca di San Lorenzo, e Pieve di Marebbe (fig. 1).

Tale campione territoriale è stato assunto preliminarmente come rappresentativo in quanto situato all' intersezione tra la Val Pusteria e la Val Badia, in un punto particolarmente interessante per la comprensione dei meccanismi del popolamento in età preistorica e protostorica (BAGOLINI \& TECCHIATI, 1993b).

L' esame dell'area geografica prescelta si è svolto lungo un tracciato viario - la "vecchia" strada della Val Badia (fig. 2) - che è da ritenersi piuttosto antico. Esso potrebbe aver veicolato anche, in tal caso, la prima fase dell'antropizzazione. Il tracciato, oggi asfaltato, segue fedelmente l' andamento delle curve di livello e si mantiene ad una quota compresa tra i 1000 e 1200 metri slm.

3) Alla ricerca di superficie e ai sondaggi in Val di Marebbe hanno preso parte, oltre allo scrivente. Patrizia Mion Tecchiati, Piero Tasca, Simone Occhi, Mariella Romeo, Elisabetta Mottes, Sandra Dellantonio, Silvano Bonetti. Carlo Zanghellini, Krizia Vettori, Maddalena Tomasi, Donatella Peloi, Hermann Seeber. Sara Comunello. Francesca Bazzanella, Elisabetta Realdon, Luisa Moser, Chiara Leveghi, Stefano Caviglioli, Maria Raffaella Caviglioli. Alvise Bagolini, Arianna Tamburrini, Alessandra Faes.

Le carotazioni sono state effettuate dalla Società Ricerche Archeologiche di Gianni Rizzi - Bressanone, su incarico della Soprintendenza Provinciale ai Beni Culturali di Bolzano, mentre le prospezioni a mezzo metal-detector sono state effettuate dal Sig. Gino Bombonato. A tutte le Istituzioni e ai partecipanti va il mio più caloroso ringraziamento.

Colgo l' occasione per esprimere inoltre il mio riconoscimento al Consiglio dell' Istitut Ladin "Micurá de Rü” per l' appoggio dimostrato alla nostra scelta di estendere l' indagine ad un territorio non direttamente "ladino" e in particolare per aver finanziato i sondaggi nel sito di Birch-Ost.

4) Ringrazio sentitamente il Signor Herwig Prinoth di Ortisei che, su incarico del Museo di Gardena, ci ha accompagnato nel corso delle nostre attività di rícognizione. Ad esse hanno partecipato inoltre Pamela Tavernarie Aldo Besola. 


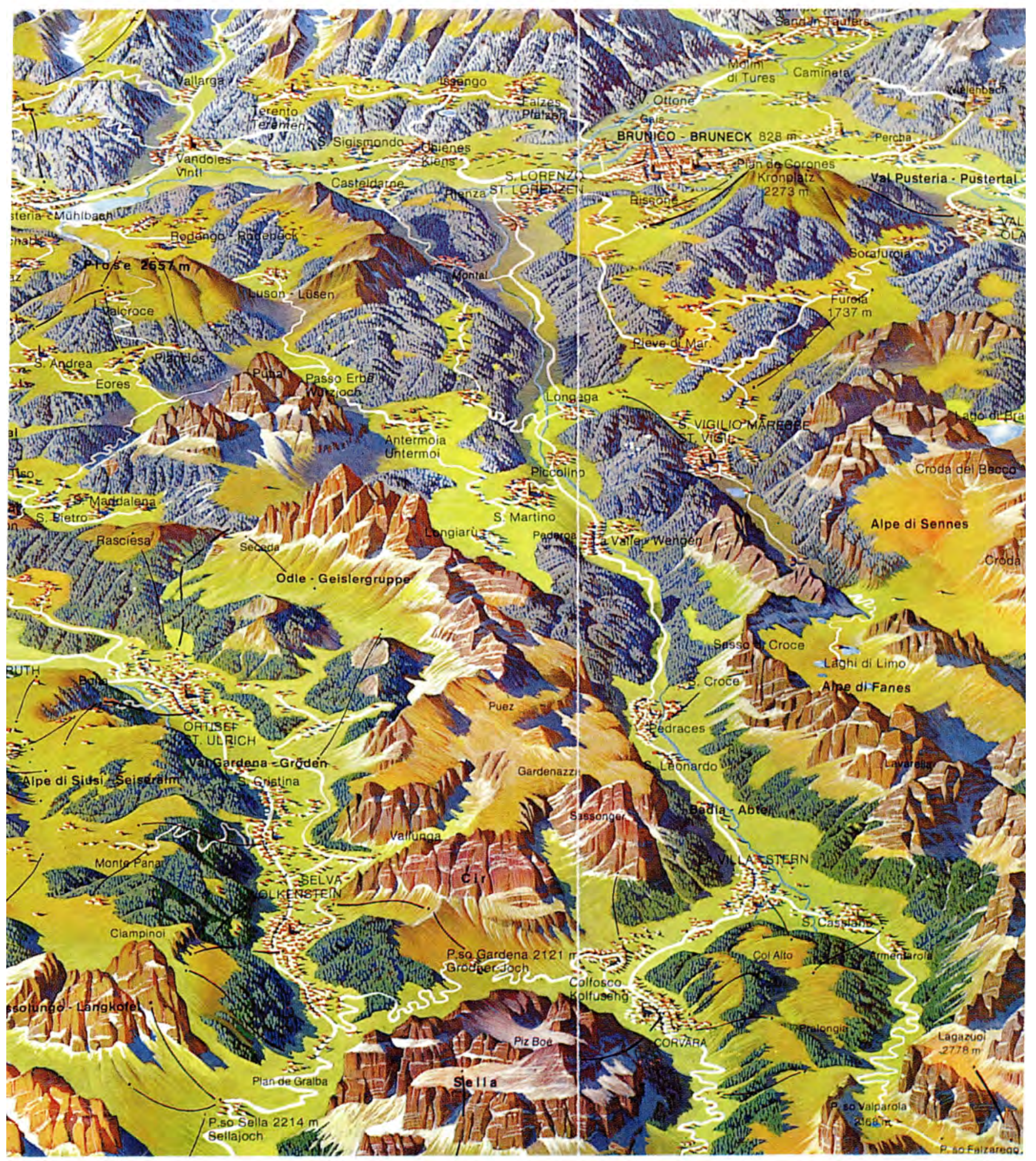

Fig. 1. Carta "a volo d'uccello" della Val Gardena e della Val Badia. La ricognizione di superficie del 1992, oggetto di questo contributo ha riguardato aree campione del circondario di Ortisei e Selva (Val Gardena) e della Val di Marebbe (bassa Badia a Sud di San Lorenzo di Sebato in Val Pusteria). 


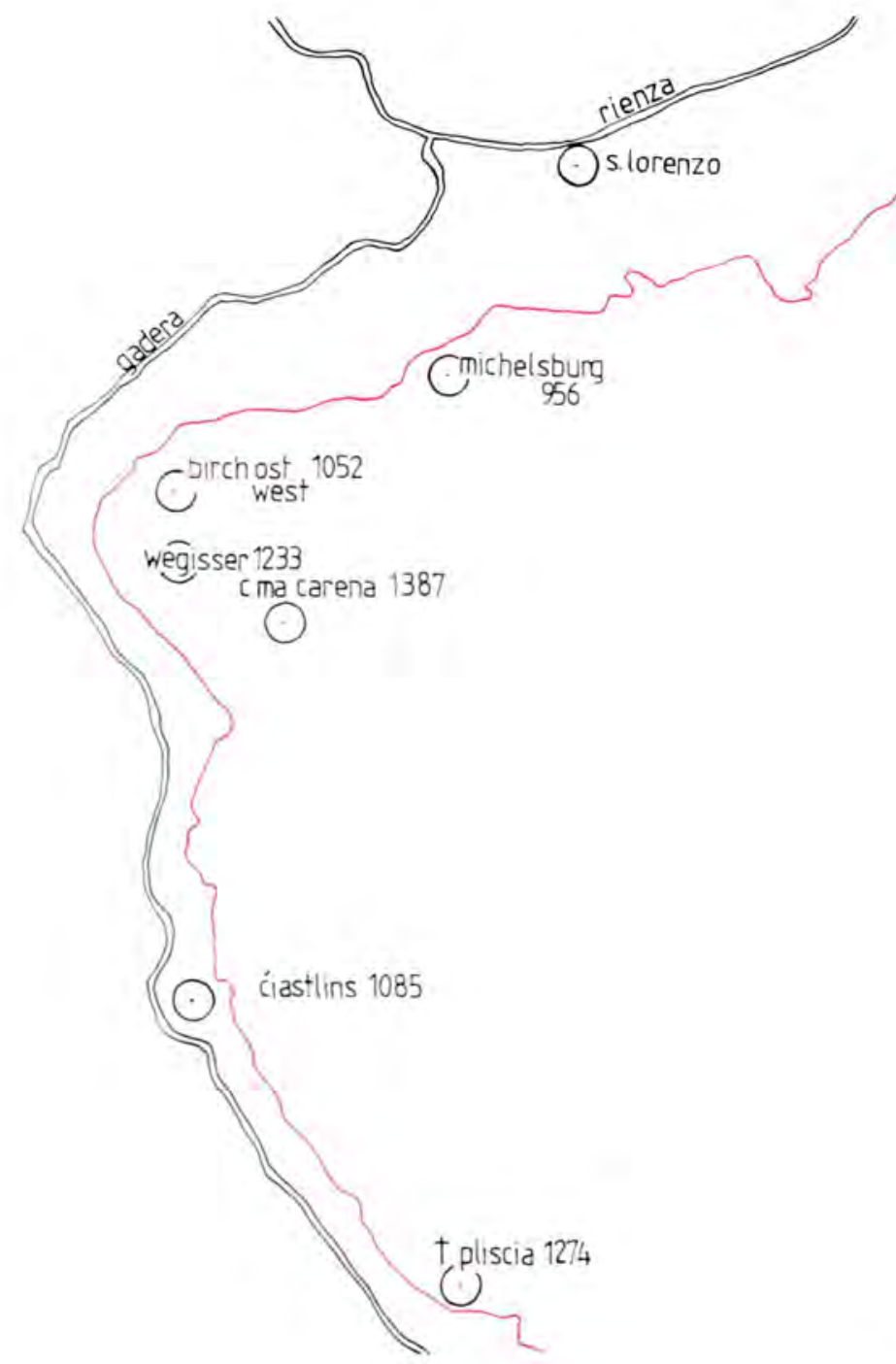

Fig. 2. La carta schematica indica il tracciato della vecchia strada della Val Badia in rapporto all'idrografia (fiume Rienza e torrente Gadera). Sono indicati i principali siti citati nel testo con le relative quote altimetriche.

L' innalzamento progressivo dipende dallo stesso progressivo innalzarsi della valle, la strada presentando la tendenza a mantenersi grosso modo in piano e ad evitare notevoli sbalzi altimetrici.

Lungo la "vecchia" strada della val Badia si situano alcuni tra i più antichi masi della zona di Marebbe, nominati nel XIII sec. nell' Urbario di Sonnenburg (Richter - Santifaller, 1937: $82 \mathrm{sgg}$.), l' abitato di Pliscia con la chiesetta costruita sulle macerie di due chiese precedenti ${ }^{5}$, l' abitato di Curt (fig. 3).

Tracciati paralleli a questo, ma altimetricamente più elevati, sono stati preliminarmente considerati come appartenenti ad un secondo, verosimilmente più recente. sistema di viabilità legato ad una ulteriore fase di antropizzazione ovvero ad un diverso impiego del territorio (silvicultura, monticazione ed economia di malga, etc.).

5) Scavi della Soprintendenza Provinciale ai Beni Culturali di Bolzano (1991). 


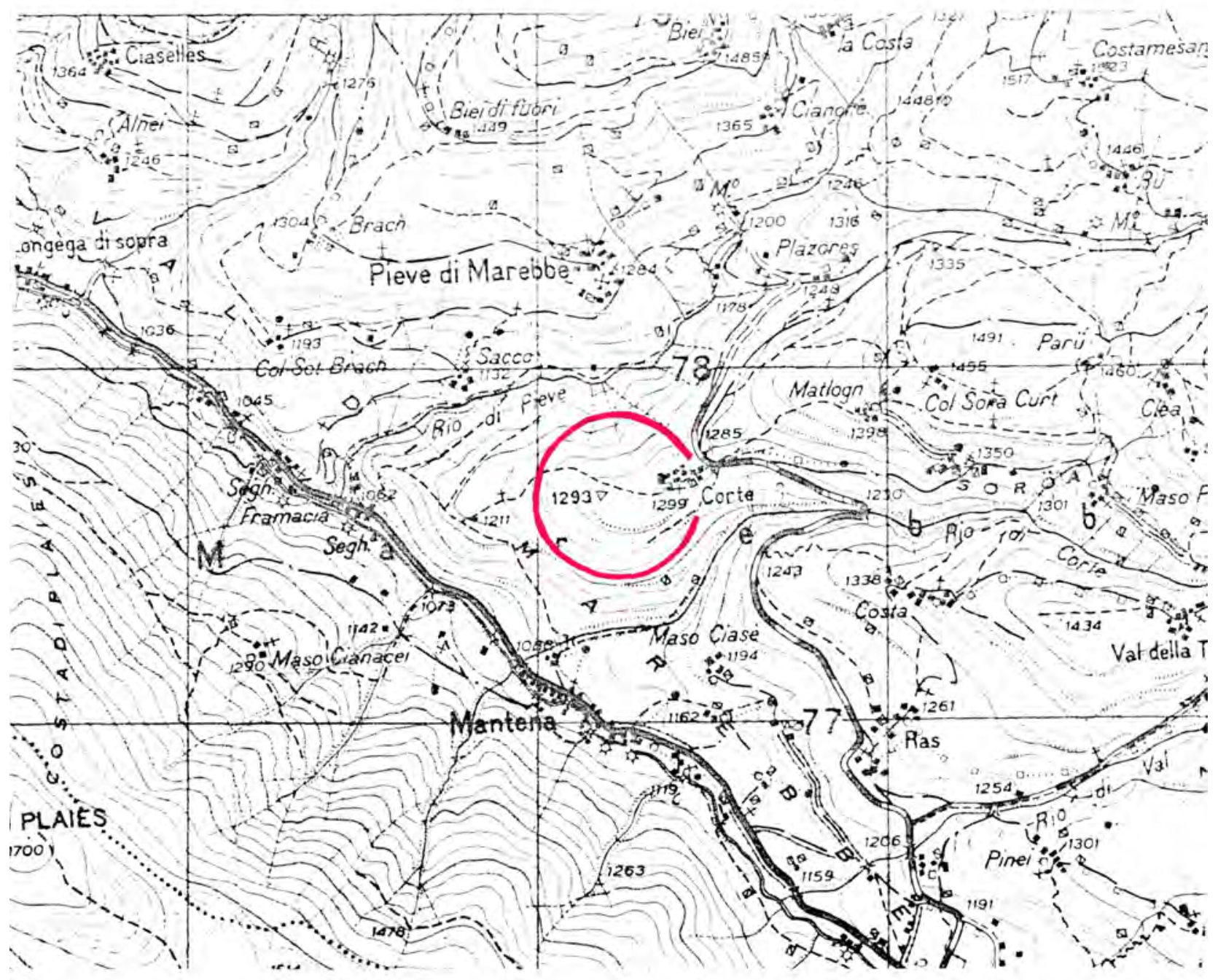

Fig. 3. L'abitato di Curt ("Corte") a Sud-Est di Pieve di Marebe si affaccia sulla vecchia strada della Val Badia. L'ampio pianoro dai lati scoscesi che si apre a ovest della chiesa e che culmina nel punto trigonometrico 1293 merita di essere ulteriormente indagato nel quadro della ricerche sul popolamento preistorico di questa porzione di Val Badia. (Riprodotto dalla Tavoletta I.G.M. 1:25.000 "Marebbe" Foglio $4^{A}$ Quadrante II, Orientamento S.E.)

Lo scopo di questa indagine consisteva nel documentare un rapporto tra possibili nuovi siti preistorici e protostorici (e comunque di interesse archeologico o significativi ai fini della ricostruzione dei diversi momenti antichi della antropizzazione) e tale tracciato originario, formulando, ove possibile, una tipologia delle preferenze insediative, spiegata in termini di utilizzo e sfruttamento del territorio in quanto parte integrante di un sistema di viabilità.

\section{1. Ćiastlins - Gschlier (Peraforada/Palfrad).}

La collina di Ćiastlins (1085 metri slm) (fig. 4) si trova nel Comune di San Lorenzo di Sebato, frazione Palù-Sares. Essa si affaccia sul corso del torrente Gadera, in posizione rilevante dal punto di vista strategico. La forma e la situa- 


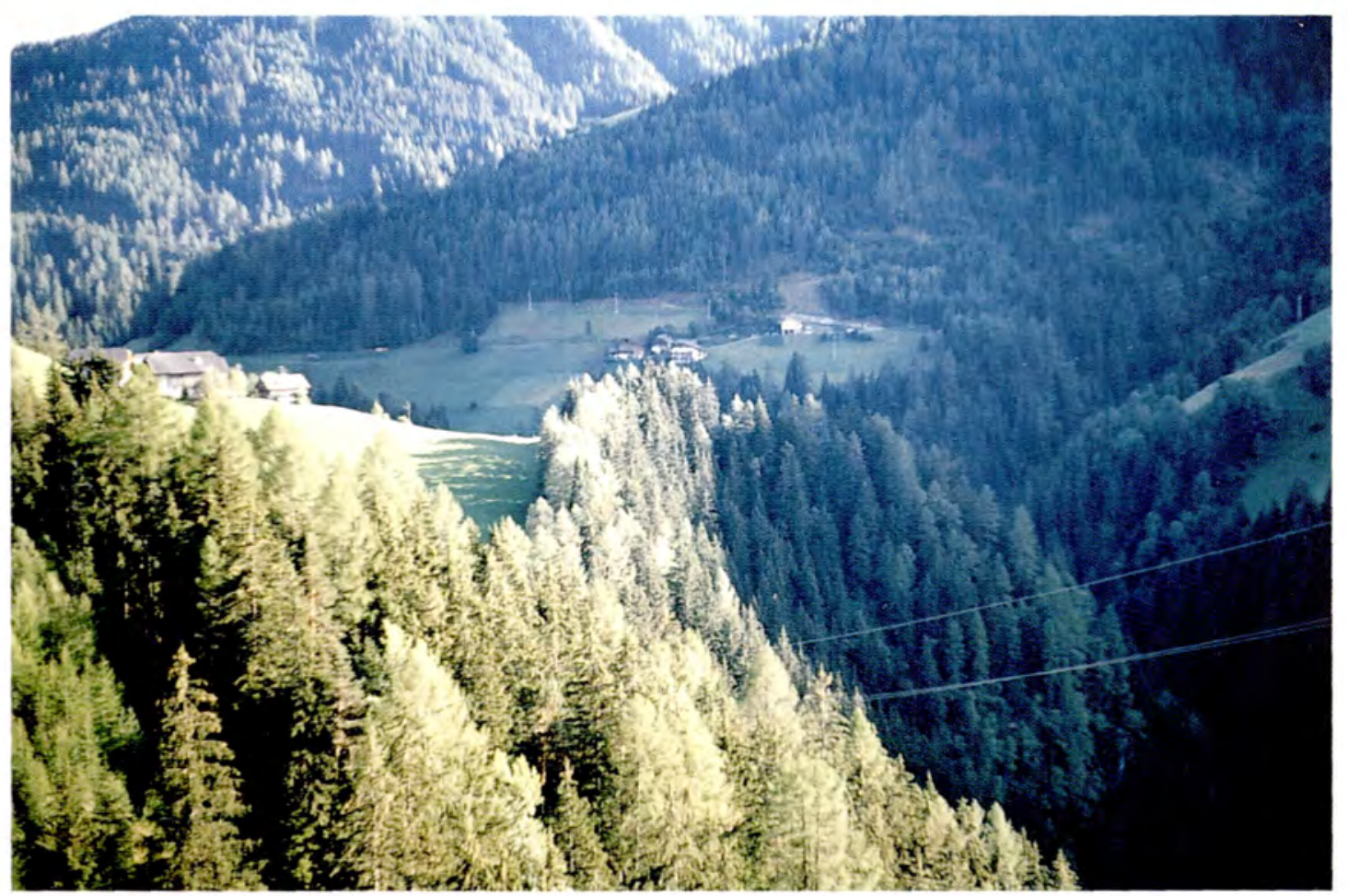

Fig. 4. Ćiastlins: Panoramica.

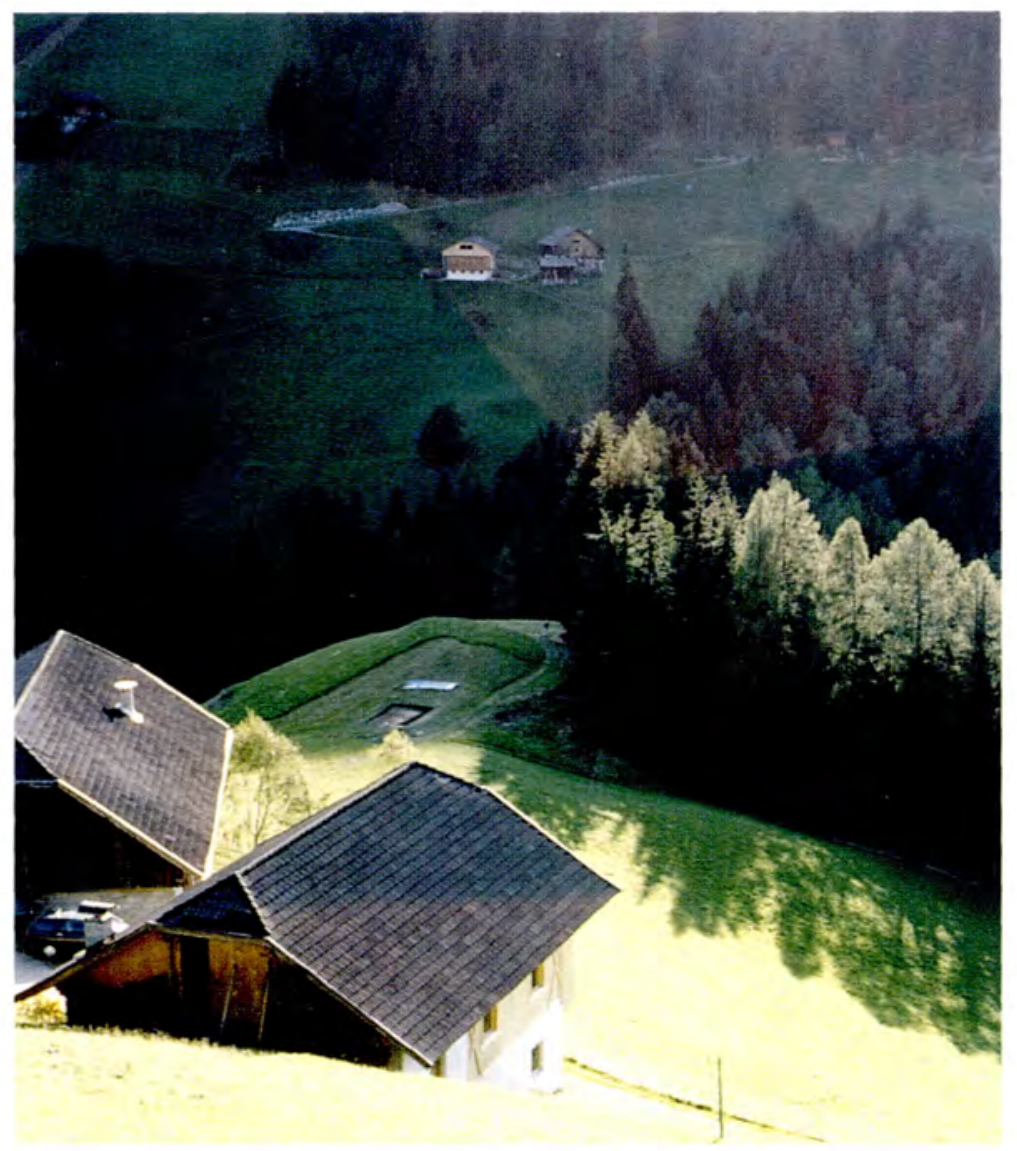

Fig. 5. Ciastlins:

Panoramica.

Al centro

dell' immagine

è visibile il saggio

di scavo (I)

aperto nella selletta

ai piedi

della sommità

del colle. 
zione complessiva ricordano l' abitato di Sotćiastel ${ }^{6)}$. Probabilmente anche per questo si sviluppò intorno ad esso un certo interesse da parte degli studiosi che si dedicarono in passato al fenomeno dei "castellieri". Tra questi ricordiamo specialmente lo scopritore, Georg Innerebner"); nel suo più recente contributo sui castellieri dell' Alto Adige, tra i quali è citato anche Ćiastlins, la cui edizione a stampa fu curata da Lunz ( cfr. nota 7), la frequentazione umana antica è datata all' età del bronzo, grazie al rinvenimento di frammenti ceramici raccolti in superficie al margine settentrionale del presunto insediamento.

Resti di strutture, parzialmente conservate, vennero osservate da Innerebner sempre sul lato nord della sommità del colle. Un secondo muro sembrò doversi individuare tra questo punto e la culminazione morfologica; esso pareva svilupparsi al di sotto della stradina che porta sul lato ovest del colle, e quindi parallelamente al pendio.

Le nostre ricerche interessarono preliminarmente la selletta tra il pendio e la sommità della collina, sul lato orientale di essa. In questo punto venne aperto un saggio di scavo misurante metri 5 sull' asse nord-sud e metri 4 sull' asse estovest, a sinistra del tratturo che scende dal gruppo di edifici del maso GschlierĆiastlins in direzione ovest, esattamente nel punto in cui il piano della selletta si alza per dare luogo al piccolo "pan di zucchero" del colle di Ćiastlins (fig. 5). Il livello agricolo-arativo (zolla), dello spessore di $\mathrm{cm} \mathrm{10-15}$ conteneva resti ceramici smaltati di età genericamente post-medioevale, la cui presenza è pertanto da considerare legata alla vita del maso. L' agricolo conteneva inoltre, in sospensione, minuti frammenti di carbone.

L' assoluta mancanza di stratificazioni e resti materiali in grado di documentare un' attività antropica antica in loco consigliò ad un certo punto di approfondire il saggio di scavo (denominato "A") solamente sul lato nord, in una fascia di terreno larga circa un metro (fig. 6). Nell' ambito di questa trincea, al di sotto dell' arativo, e per una profondità di metri 0,50 circa, è stato scavato uno strato di terreno bruno chiaro, definibile subagricolo, contenente frequenti carboni in sospensione, ma assolutamente privo di resti antropici sia antichi che moderni. Alla base di questo strato sono stati osservati numerosi clasti di asse compreso tra $\mathrm{cm} 20$ e 40, apparentemente giustapposti, ma quasi sicuramente non strutturati (fig. 7), che riteniamo pertinenti al sostrato morenico sterile di fondo.

Contestualmente è stato avviato un saggio di scavo (saggio "B") in corrispondenza del muro a secco sul lato nord della sommità della collina, che in un primo momento si era sospettato antico (fig. 8), ma che deve invece essere stato eretto in tempi recenti a sostegno del margine di valle del tratturo, in quel punto particolarmente ripido e soggetto a smottare.

Nell' ambito della sezione occasionale aperta dal proprietario del fondo, Signor Innerhofer, pochi metri ad est del "muro" a secco, nel corso della pulizia

6) Lunz, 1979, Bagolini, Tasca \& Tecchiati, 1989 e 1990-91; Tecchiati, 1990. Dragogna, Tecchiati \& Walzolgher,
1991. Bagolini \& Tecchiati, 1993 b.

7) cfr. Innerebner, 1956, 1961, 1975. 


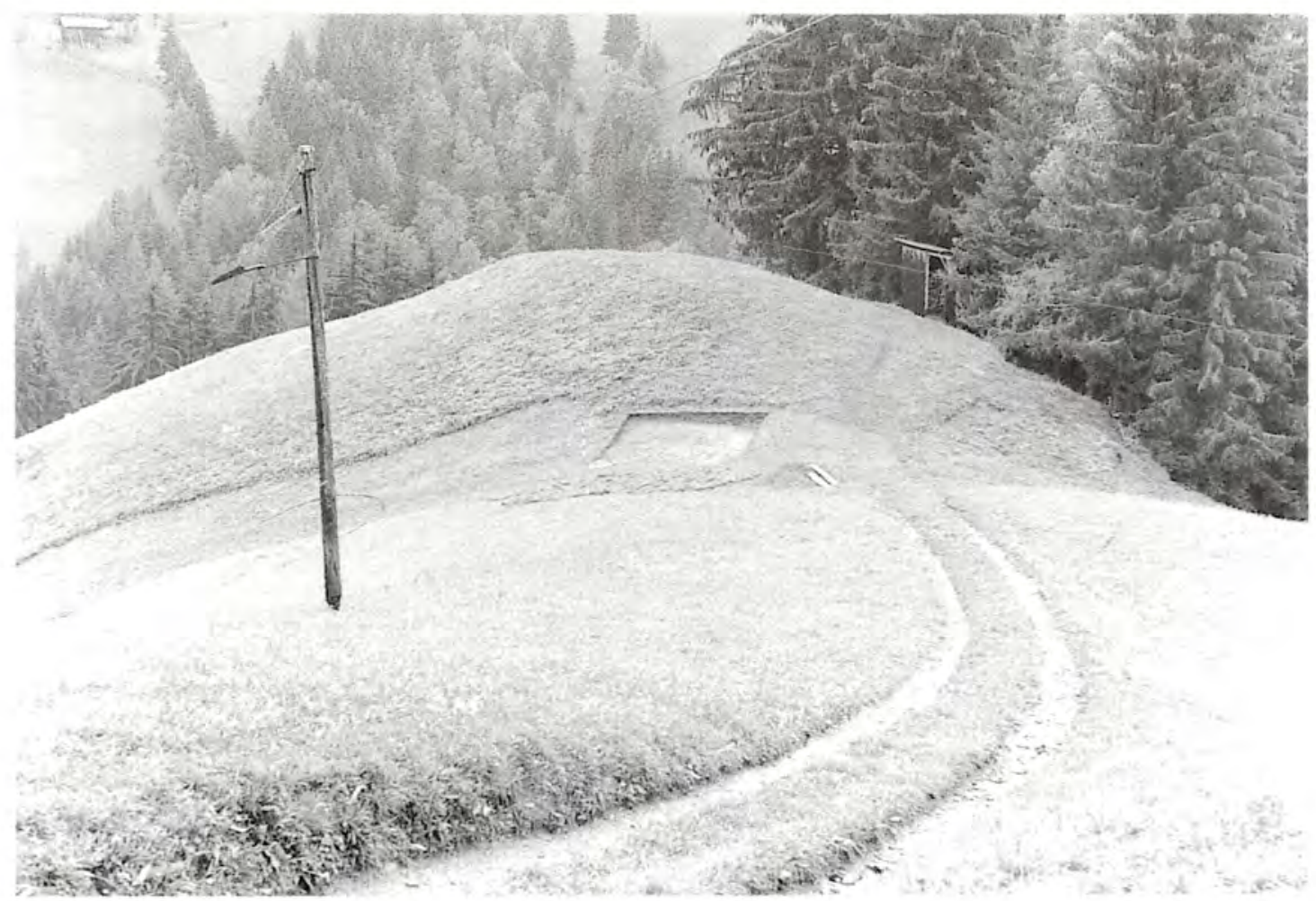

Fig. 6. Ćiastlins: Al centro della foto, il saggio " $A$ ".

Fig. 7. Ciastlins: Dettagli del saggio "A". A destra nella foto (lato Nord) la trincea di assaggio approfondita fino allo sterilé.

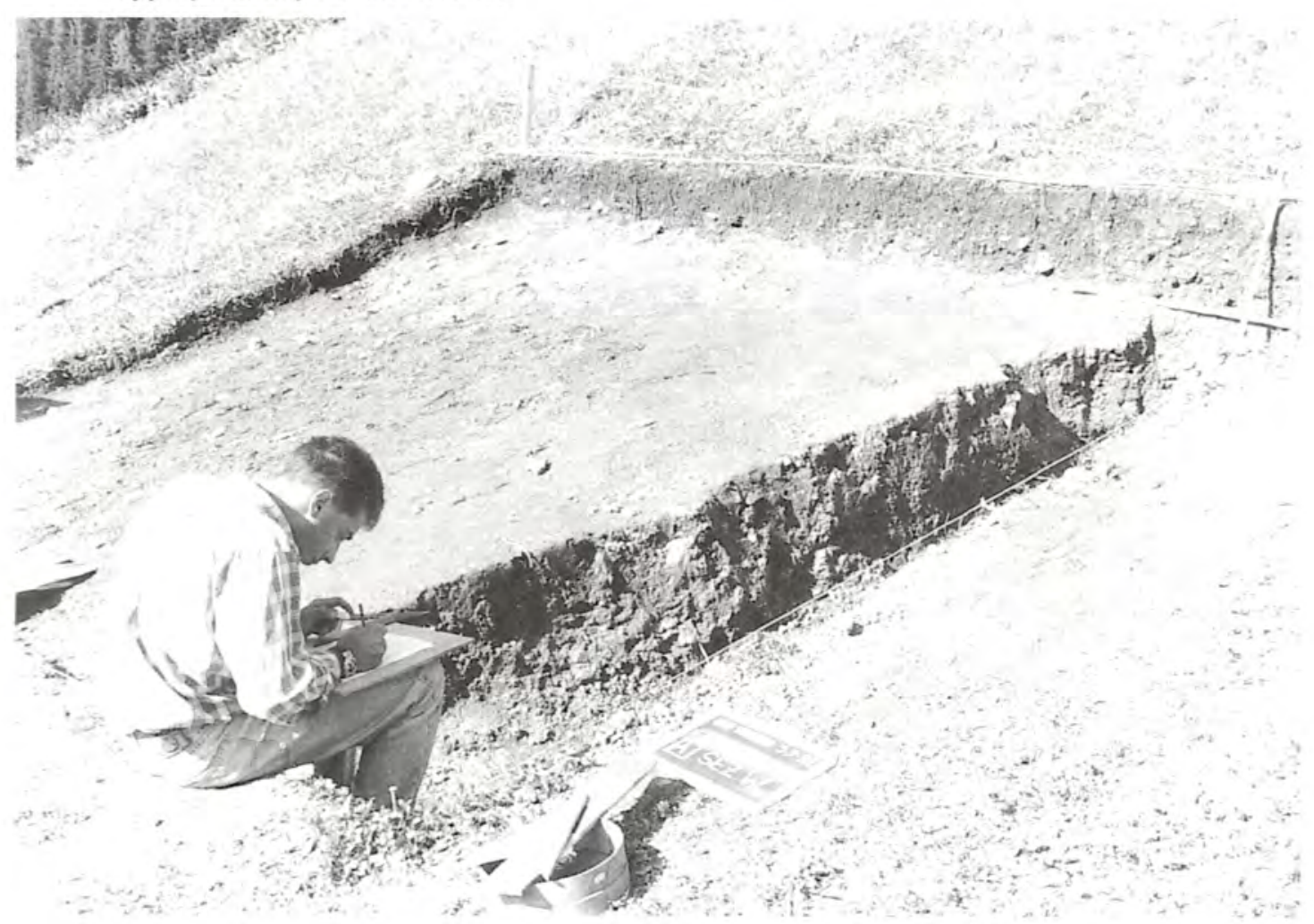




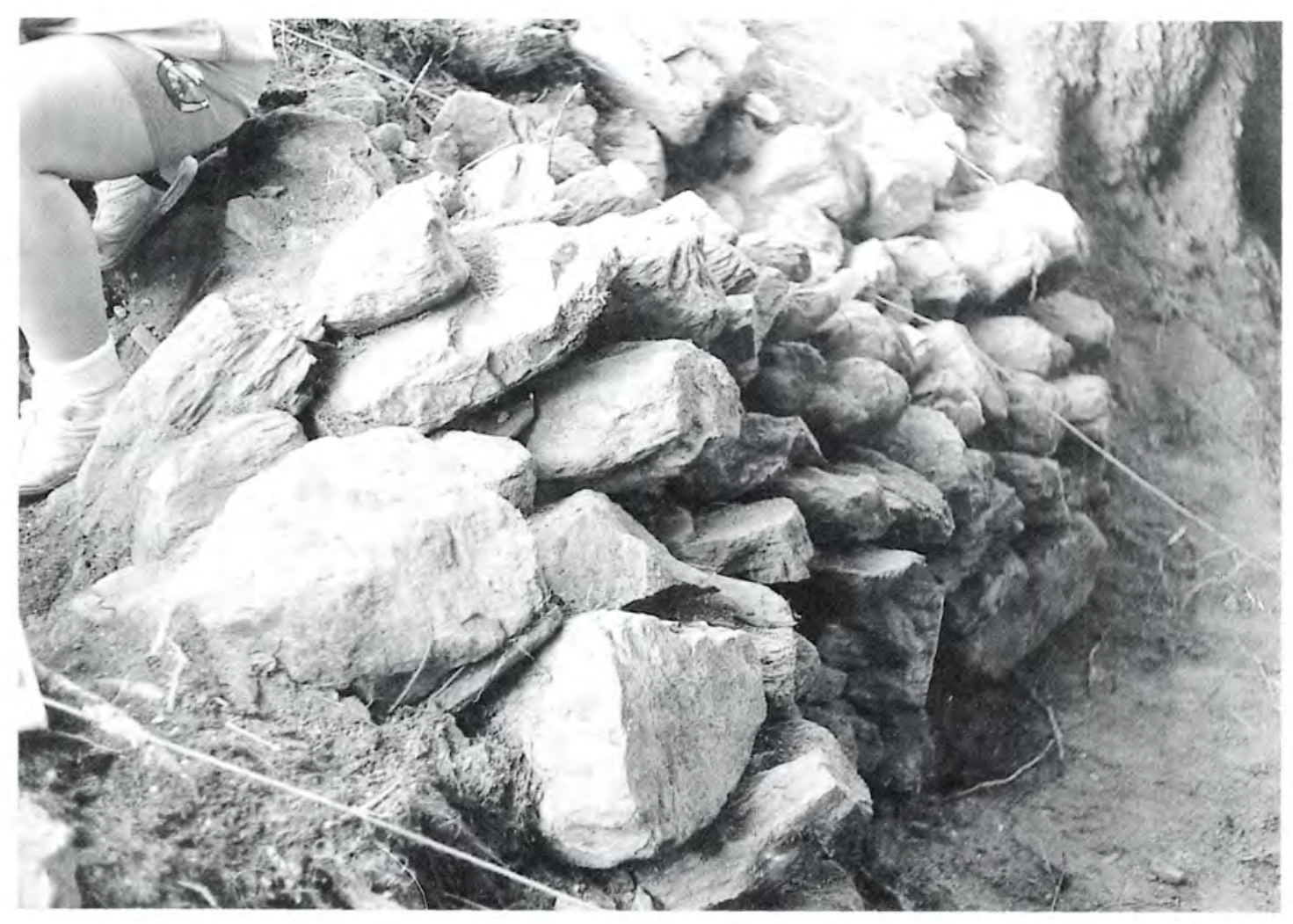

Fig. 8. Ciastlins: Dettaglio di scorcio (a) e di prospetto (b) del muretto a secco sul lato Nord della sommità della collina. Nessun dato archeologico concreto per qualificarlo antico.

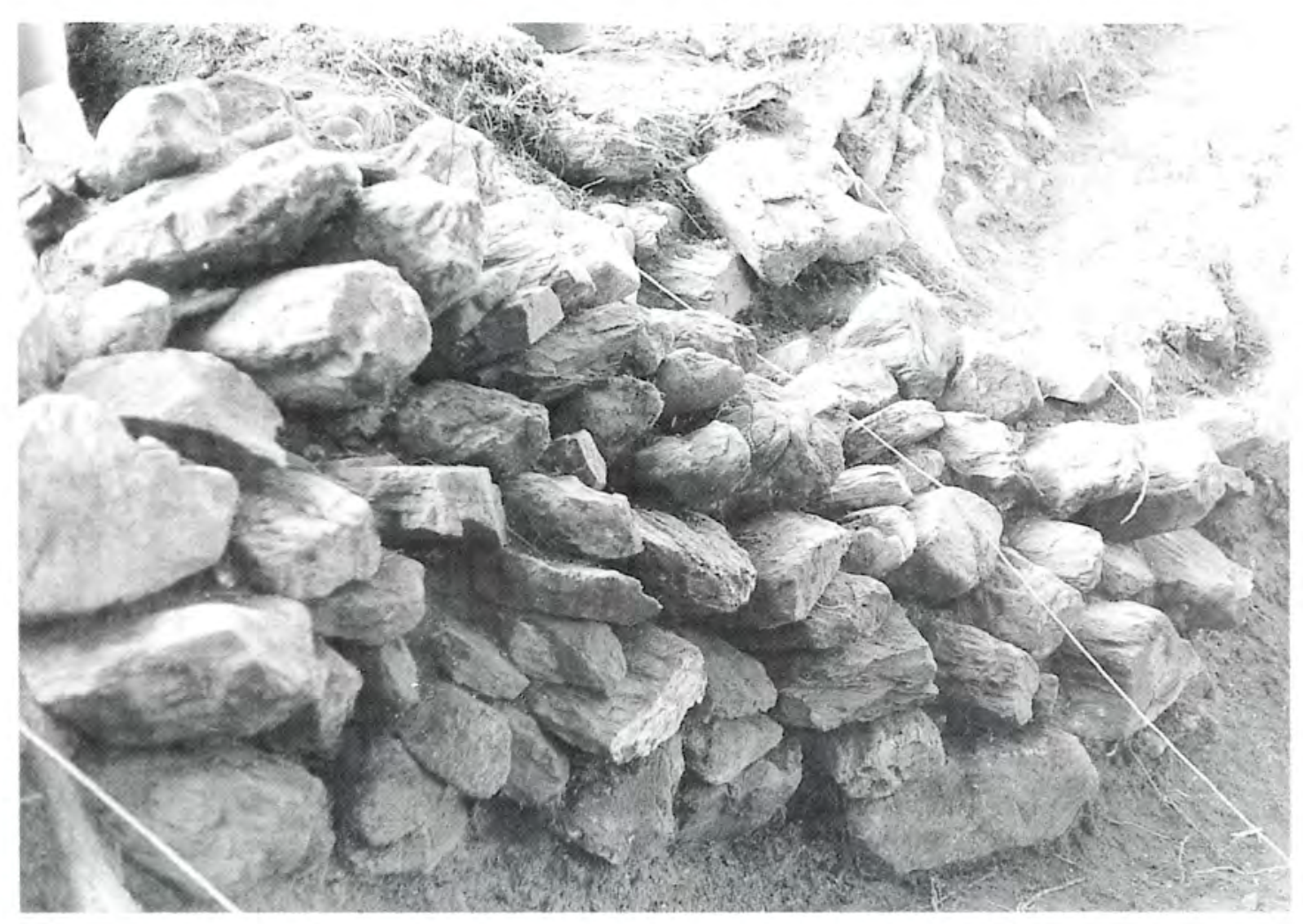



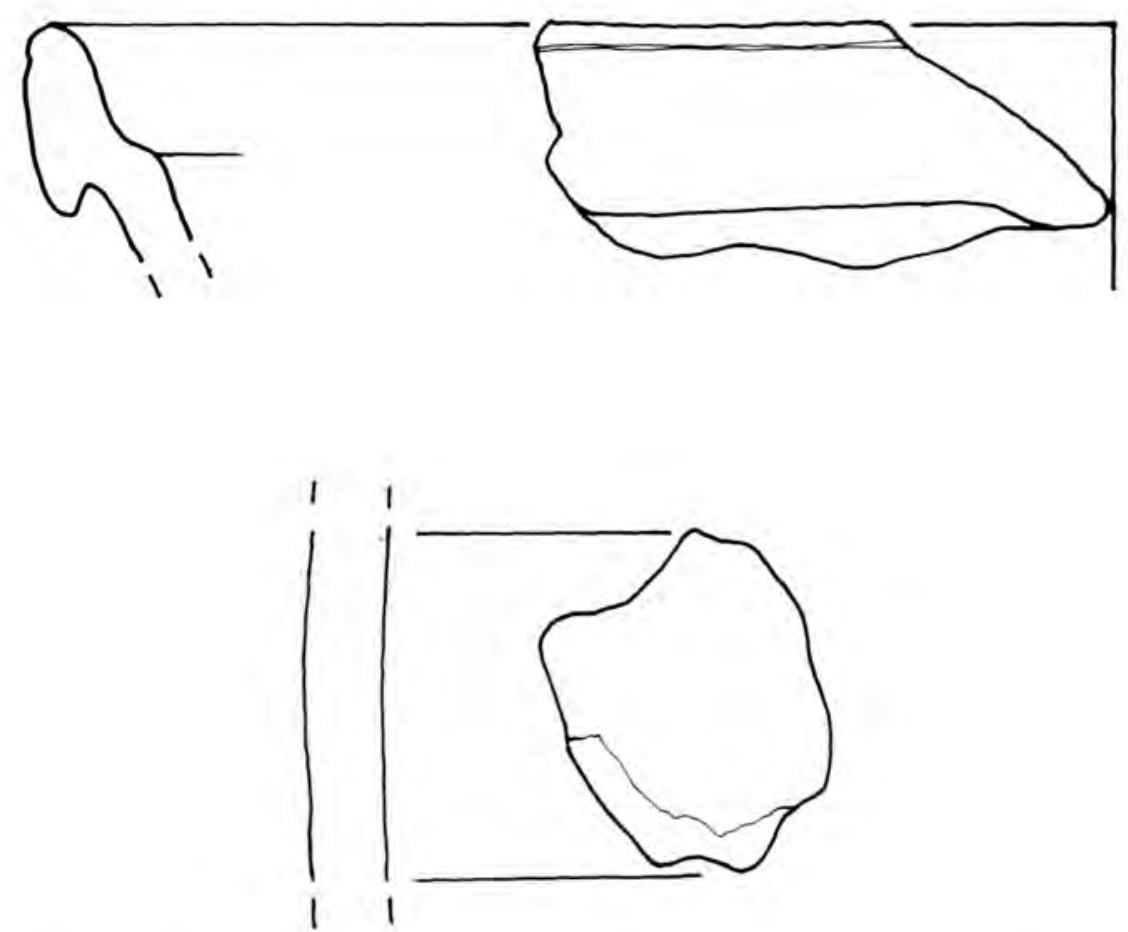

Fig. 9. Ciastlins: Frammenti ceramici della sezione occasionale Nord. Il frammento atipico in basso è certamente preistorico.

della medesima sono venuti alla luce, provenienti da un livello chiaramente rimescolato, due frammenti ceramici, uno dei quali sicuramente preistorico, l' altro invece pertinente ad un recipiente affine al tipo "Passau" di età presumibilmente basso- o postmedioevale (fig. 9).

Un ulteriore saggio (denominato " $\mathrm{C}$ ") sul pendio che dà sul sentierino che si stacca dal tratturo in direzione nord, all' incirca all' altezza del saggio " $\mathrm{A}$ " e ad una decina di metri a destra di questo, non portò alcun dato nuovo, se non pietrame sciolto non strutturato (effetto forse di dissodamenti recenti) e terreno rimescolato caratterizzati dalla completa assenza di resti antropici sia antichi che moderni.

Prove con il metal-detector nell' area a ovest del maso e delle adiacenze delle aree da noi sottoposte a scavo hanno fruttato alcune lamine di bronzo, di cui una decorata con sottili linee incise, di ardua attribuzione cronologica, ed un anello di bronzo, forse in origine dorato, presentante tre pietre, certamente non molto antico (fig. 10).

L'area della collina è stata sottoposta inoltre a prospezioni a mezzo carotatrice. Numerose prove effettuate in svariati punti della collina di Ćiastlins, e in prevalenza lungo il margine nord-occidentale, ma anche sul versante sud e sulla sommità della medesima, non hanno fornito alcun dato archeologicamente rilevante; esse hanno evidenziato, al di sotto dell' agricolo, un terreno sciolto sabbioso-ghiaioso molto incoerente, caratterizzato da ghiaia grossolana a vuoti, in tutti i casi privo di resti archeologici. 

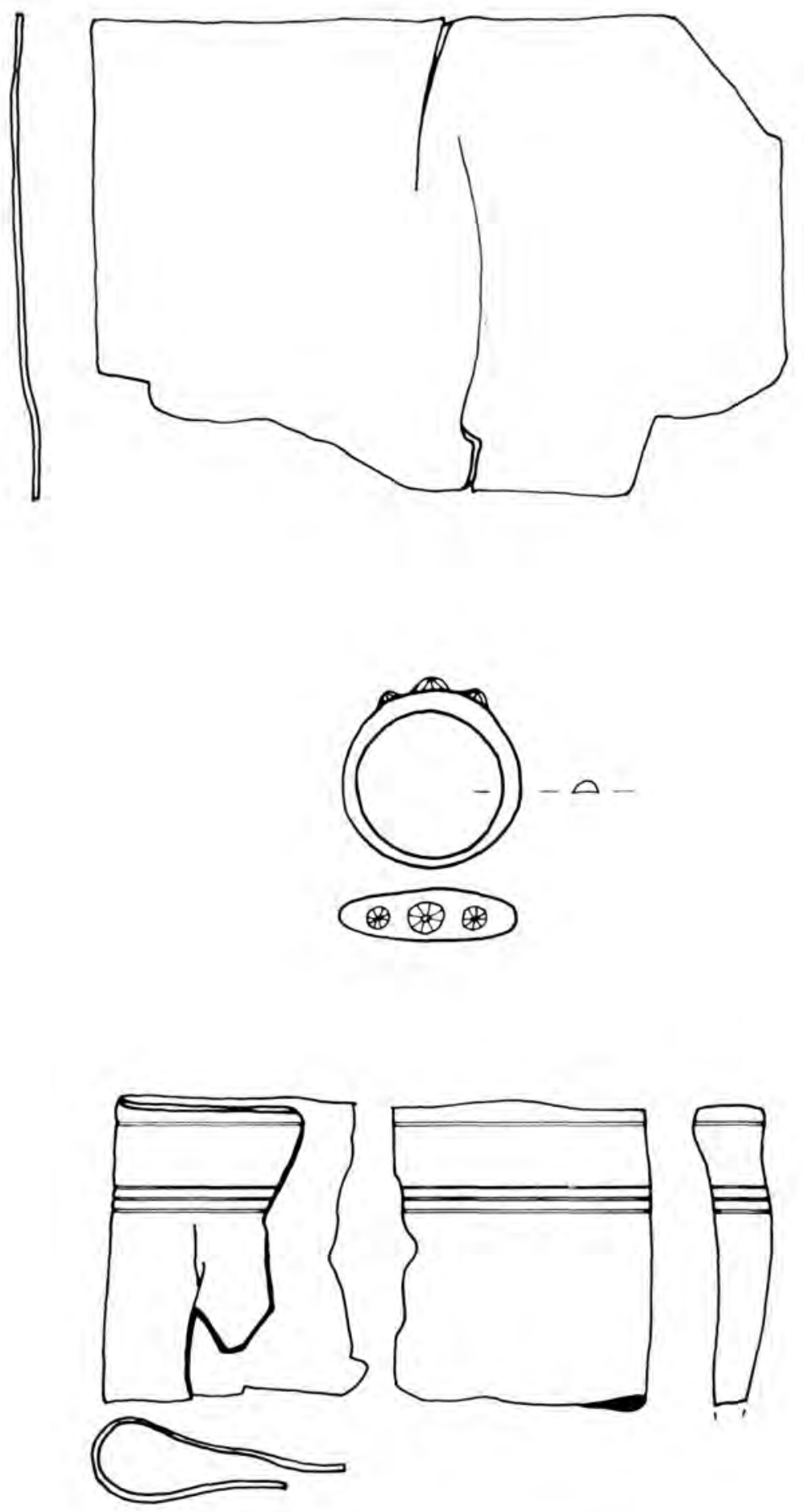

Fig. 10. Ćiastlins: Reperti metallici dalle prospezioni a mezzo metal-detector. 
I pochissimi dati raccolti non hanno permesso di fornire un inquadramento cronologico e culturale, ed un significato "strategico" ad un eventuale insediamento antico.

Ciononostante alcuni elementi - fra i quali il toponimo stesso, generalmente considerato indicatore di siti pre- o protostorici - suggeriscono di non demordere dal riservare a questo piccolo sito d' altura quell' interesse che le prove documentarie non hanno potuto finora avallare.

Ai pochi resti ceramici citati da Innerebner, e al solo coccio preistorico rinvenuto nella campagna 1992 non si può attribuire quasi alcun significato. Peraltro, una eventuale presenza antropica antica di età preistorica o protostorica potrebbe anche non essersi configurata in senso specificamente insediativo; già altrove (BAGOLINI \& TECCHIATI, 1993b) si è voluto sottolineare come non necessariamente la frequentazione di un sito (per es. a scopo "militare", o per temporaneo rifugio, o per attività connesse alla custodia di greggi entro recinti lignei o altro) lasci resti rilevabili in sede archeologica ${ }^{8)}$.

\section{2. Pieve di Marebbe - località "Curt".}

Proprio dirimpetto alla chiesa di Curt, lungo una stradina sterrata su cui si affacciano i masi, sono stati rinvenuti almeno due livelli carboniosi distinti, che appaiono chiaramente in sezione (la stradina sterrata ha tagliato un piccolo rilievo, alto sul fondo stradale un metro circa): nessun rinvenimento archeologico. Tale piccolo rilievo sembra almeno in parte prodotto da strutture murarie sepolte pertinenti a quanto pare ad un antico edificio (fienile?) distrutto dal fuoco e ricostruito altrove. I resti murari si sviluppano, per quanto è possibile determinare sulla base dei tratti visibili in superficie, per una lunghezza di $\mathrm{m} 8$ 10 e per una larghezza, parallelamente alla stradina sterrata, di m 10-15 circa.

\section{3. San Vigilio di Marebbe - località "Costa".}

E' stato effettuato il controllo dei numerosi sbancamenti notati lungo la strada che da Pieve di Marebbe porta a Ćiastlins, effettuati per l' allargamento di alcuni tratti particolarmente angusti della medesima. Le incisioni operate nel pendio a mezzo escavatore meccanico hanno evidenziato in tutti i casi un sottile strato agricolo limitato alla zolla, impostato direttamente sul suolo vergine in posto, non interessato da stratificazioni antropiche e privo di resti archeologici.

In località "Costa", subito ad est di San Vigilio di Marebbe, nell' ambito di un cantiere edile, ci si è dedicati al rilievo e alle campionature di una serie di livelli in parte carboniosi provenienti da una sezione occasionale (fig. 11 e fig. 12).

8) Considerazioni analoghe devono essere espresse anche, a quanto pare, per il presunto "abitato" di Birch-Ost, per il quale cfr., infra, il par. 1.4. 

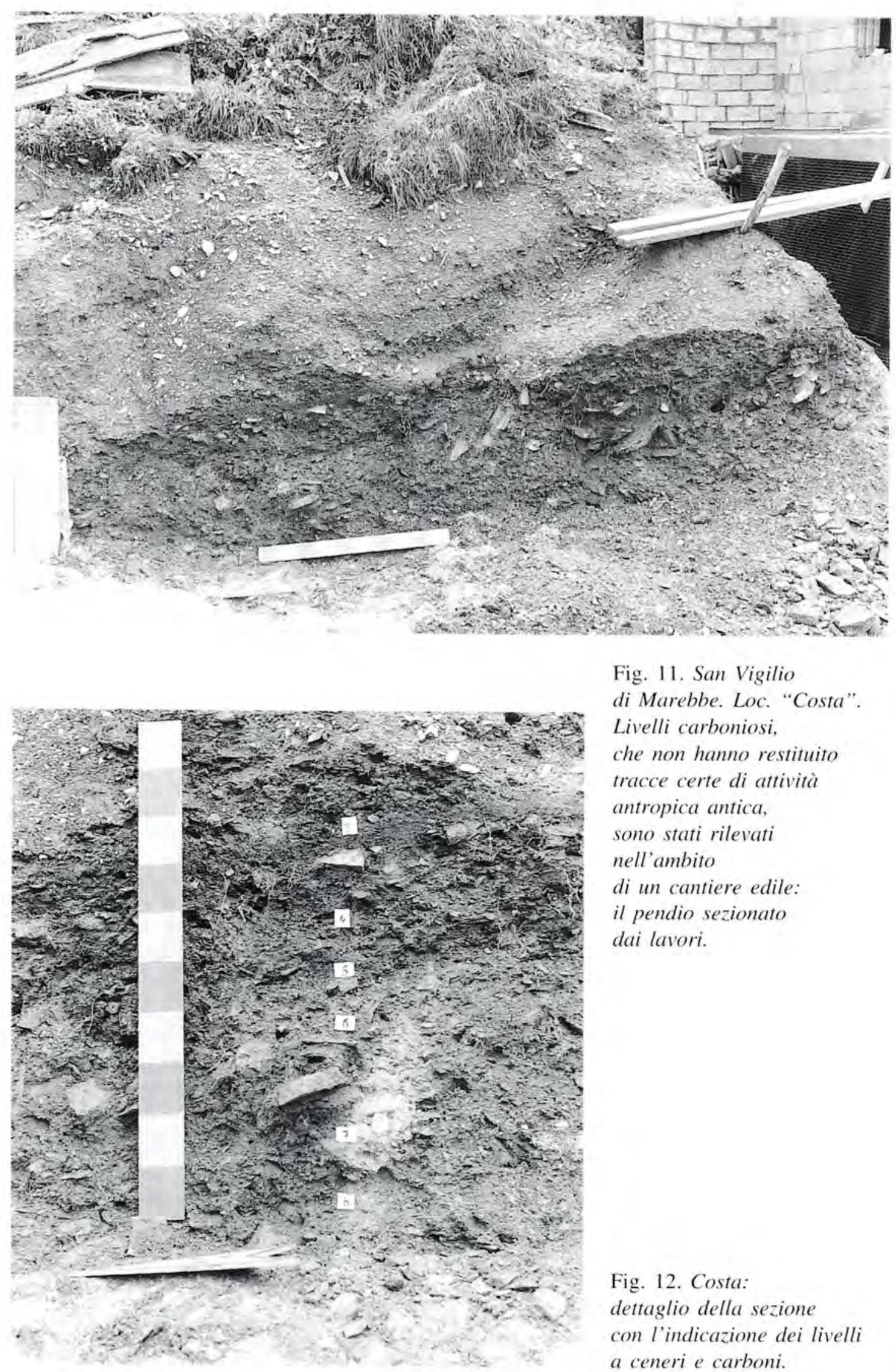

Fig. 11. San Vigilio di Marebbe. Loc. "Costa". Livelli carboniosi, che non hanno restituito tracce certe di attività antropica antica, sono stati rilevati nell'ambito di un cantiere edile: il pendio sezionato dai lavori.

Fig. 12. Costa:

dettaglio della sezione con l'indicazione dei livelli a ceneri e carboni. 
Ad un metro circa di profondità dal suolo attuale, in una situazione di leggero pendio, si è notato un livello scuro a marcata componente argillosa che si sviluppa per una lunghezza di metri quattro circa, potente all' incirca $\mathrm{cm} 5$; ben al di sotto di questo strato, che potrebbe configurarsi come un antico (?) piano di calpestio o paleosuolo vegetale, si è notata una lente di terreno carbonioso contenente frammenti di carbone anche centimetrici. Essa misura $\mathrm{cm} 40 \mathrm{di}$ lunghezza; la sua potenza si aggira sui $\mathrm{cm} 5$ al centro, e sfuma verso i margini. $\mathrm{Al}$ di sotto di questo strato l' argilla (paleo-humus di bosco) è apparsa molto ossidata. Attesa l' assenza di reperti antropici la sezione occasionale non è stata disegnata ma soltanto fotografata.

\section{4. Birch-Ost e dintorni.}

$\mathrm{L}^{\prime}$ indagine territoriale ha riguardato anche la zona della chiesetta di Santa Maria di Saalen-Sares all' inizio della Val Badia. La parte orientale dell' area è dominata da almeno quattro rilievi morfologicamente interessanti: Colle Wegisser, Cima Carena, Birch-Ost e Birch-West. Le prime due culminazioni sono risultate fittamente ricoperte da vegetazione a conifere; per tale ragione non è stato possibile, mediante la sola analisi di superficie, individuare situazioni di interesse archeologico. $\mathrm{Ci}$ sentiamo però di considerare probabile una loro antropizzazione nella pre-protostoria, a giudicare dalla morfologia e dalla prossimità alla viabilità tradizionale.

La sommità del Colle Birch-West si presenta sviluppata piuttosto in lunghezza che in larghezza, con maggiore estensione lungo l' asse approssimativo $\mathrm{N}$-S. Essa è molto accidentata per la presenza di molte pietre in assetto caotico che rendono assai ardua l' analisi di superficie. L' impressione generale è che questo pietrame, soprattutto quello di pezzatura maggiore $(30-60 \mathrm{~cm}$ di asse e oltre) possa essere riferito a strutture collassate. Il tentativo di fornire una probabile cronologia a queste presunte strutture, mediante limitati saggi di scavo in aree considerate favorevoli, non ha fornito alcun dato probante. Appare però plausibile che il colle sia stato frequentato nell' antichità e che abbia ospitato un insediamento del tipo "su altura". Va osservato che tale supposizione potrebbe essere preliminarmente confortata dal rapporto intercorrente tra questa culminazione e il sistema viario antico o tradizionale.

La sommità del Birch-Ost (fig. 13), perfettamente pianeggiante, raccordata al Birch-West per mezzo di una dolce selletta che si apre in direzione S-W, appare caratterizzata da un muro di cinta a pianta irregolarmente peniforme, $\mathrm{i}$ cui assi misurano approssimativamente $\mathrm{m} 14$ per 42 . In alcuni punti tale muro presenta allungata ancora una certa sopraelevazione rispetto al suolo attuale, e conci squadrati posti in opera a secco con una qualche accuratezza.

9) Al di là dell' esito negativo di questi controlli, se ne deve ancora una volta rilevare I' importanza nel quadro della imprescindibile necessità di presidio del territorio ai fini di tutela, a fronte di una attività edilizia e agricola che ri- schia ogni giorno di compromettere situazioni archeologiche sconosciute e tanto più preziose in un areale le cui potenzialità archeologiche principiano soltanto ora a palesarsi nella loro reale entità. 
Fig. 13 .

Panoramica da Ovest del Colle Birch-Est.

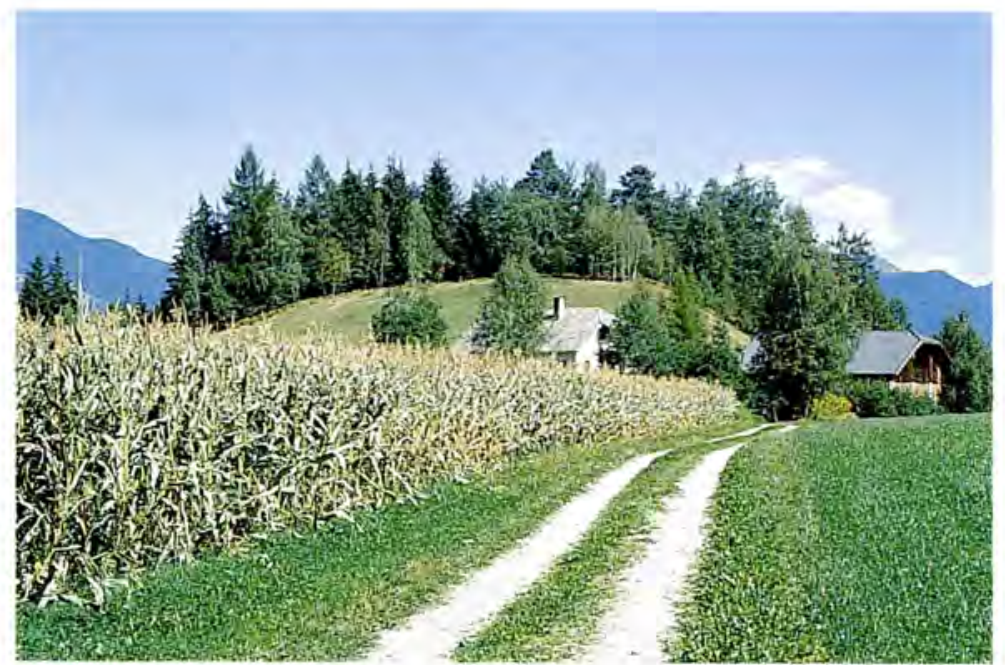

Sei saggi di scavo (figg. 14-17) di limitata estensione sono stati praticati sul muro di cinta e al centro dell' area da questo racchiusa.

Parte di questi sondaggi sono stati finalizzati alla messa a nudo della faccia interna del muro. Sul lato $\mathrm{N}-\mathrm{W}$ se ne è messa in luce anche la faccia esterna (saggio III).

Il muro è costituito da grossi conci squadrati, che si dispongono per lo più in doppia fila accostata: ciascuna di queste file dà generalmente luogo ad una "faccia interna" e ad una "faccia esterna" del muro. In alcuni casi pietre di grandi dimensioni disposte trasversalmente per l' intera larghezza del muro (come per es. nel saggio III) sembrano rivestire una funzione di "cerniera" tra i due allineamenti paralleli, evidentemente non molto coerenti tra di loro e di tenuta presumibilmente non molto elevata. Il muro si presenta conservato fino ad un' altezza massima di m 0,50 circa rispetto al suolo di calpestio attuale; in alcuni punti si presenta lacunoso forse a seguito di spoliazioni più o meno recenti. La larghezza del manufatto è variabile e difficile da determinare a causa dei crolli: nei punti indagati essa è risultata variabile tra $\mathrm{m} 1 \mathrm{e} \mathrm{m} \mathrm{1,50}$ circa.

Preliminarmente si può osservare che la profondità del muro è maggiore all' interno che all' esterno. Tale circostanza induce a ritenere che i conci interni del muro siano stati alloggiati in un taglio artificiale, ovvero sfruttando forse la naturale leggera concavità della sommità (fig. 18), e che solo da una certa altezza in poi ai conci interni del muro siano stati affiancati i conci esterni. In altri casi, come per esempio nel saggio II, la sovrapposizione dei conci era minima sia all' interno che all' esterno: un solo ordine di conci (due file accostate) poggiante direttamente sul suolo vergine, apparentemente non alloggiato in una fossa di fondazione scavata a spese del medesimo.

$E^{\prime}$ del tutto verosimile che questa struttura, di cui si fornisce qui una pianta schematica (fig. 19), abbia subito, come detto, numerose spoliazioni nel corso dei secoli. Non è privo di significato il fatto che il muro si presenti maggiormente 
Fig. 14.

Birch Est.

La parte sommitale

del muro di cinta

messa in luce dal saggio III.

Fig. 15.

Birch Est.

Il saggio III ha riguardato anche la faccia esterna del muro di cinta,

superstite per circa

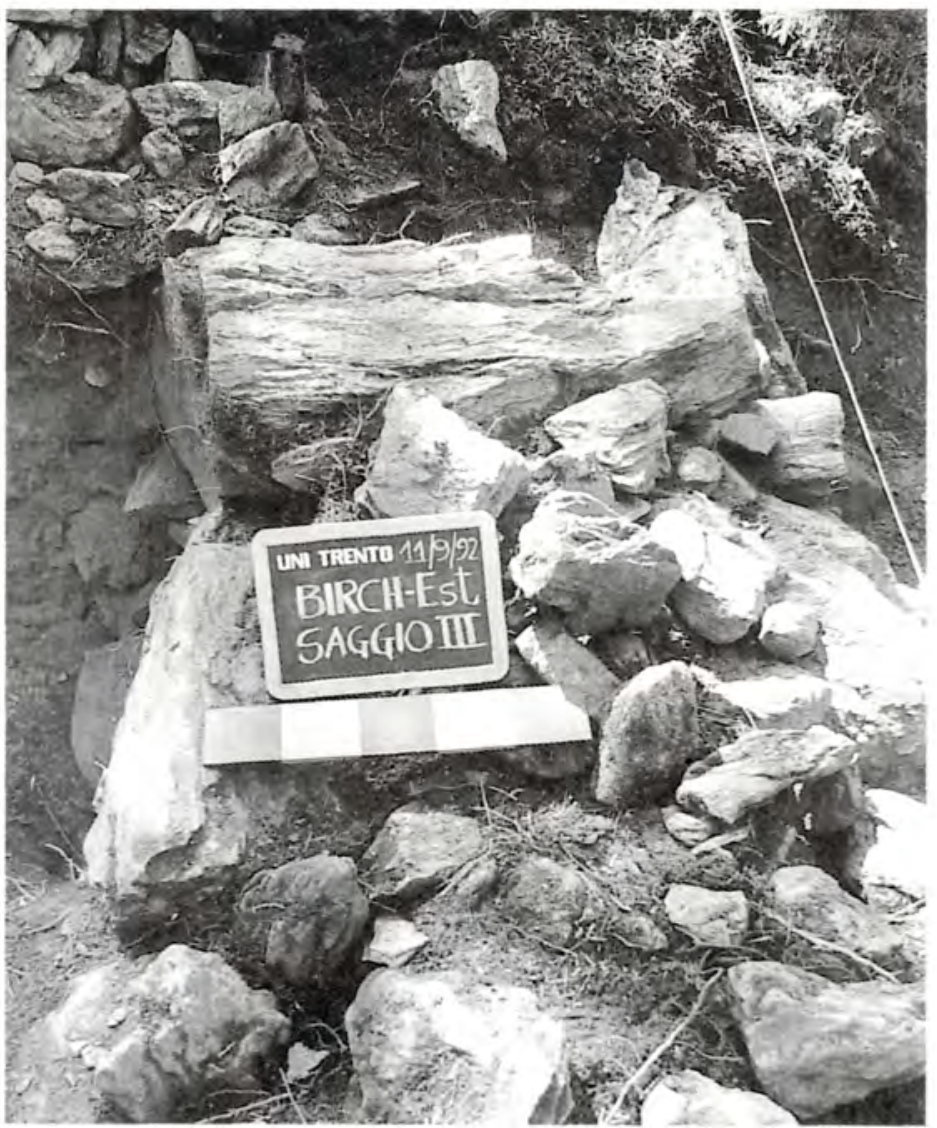
un metro di altezza.

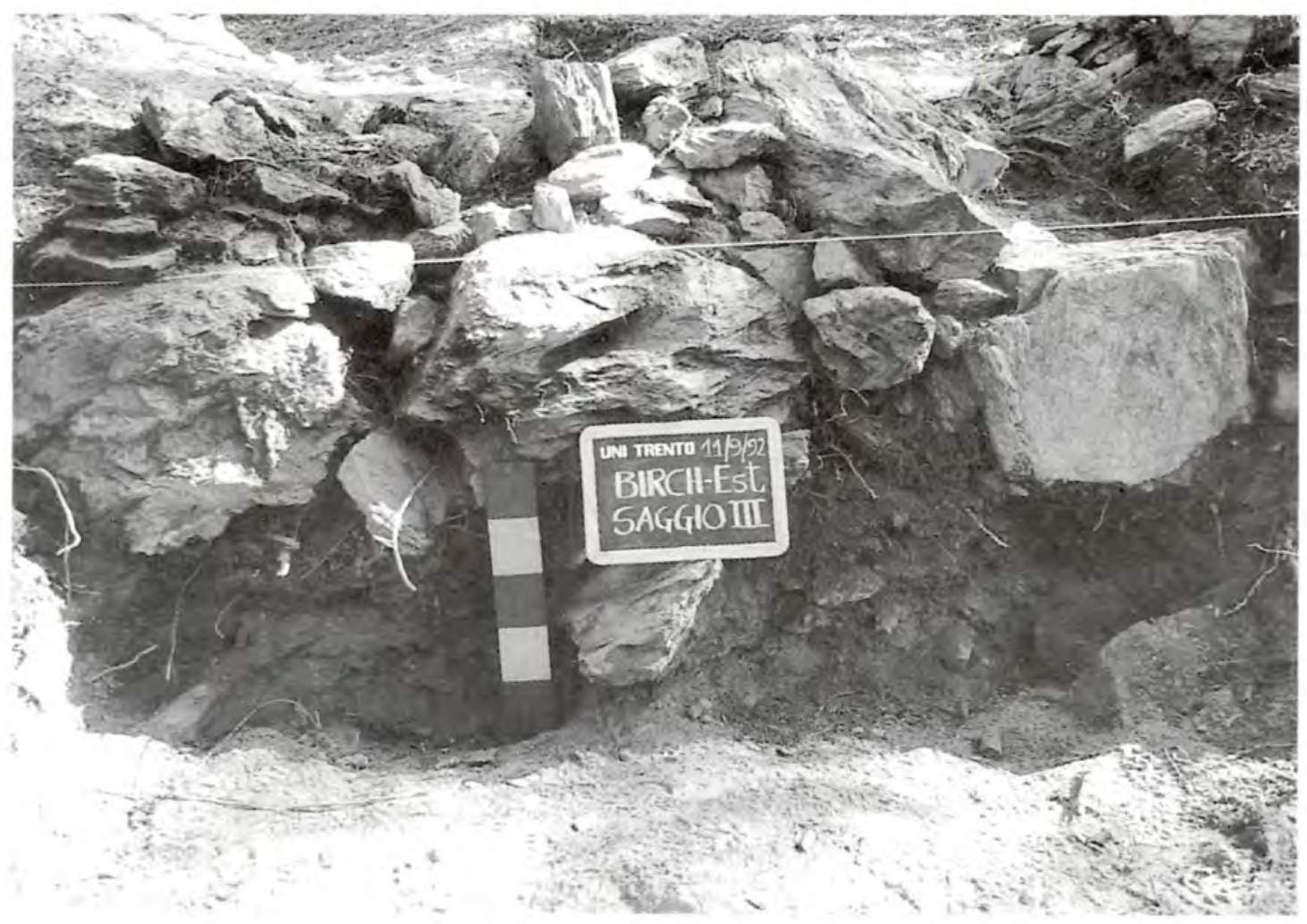




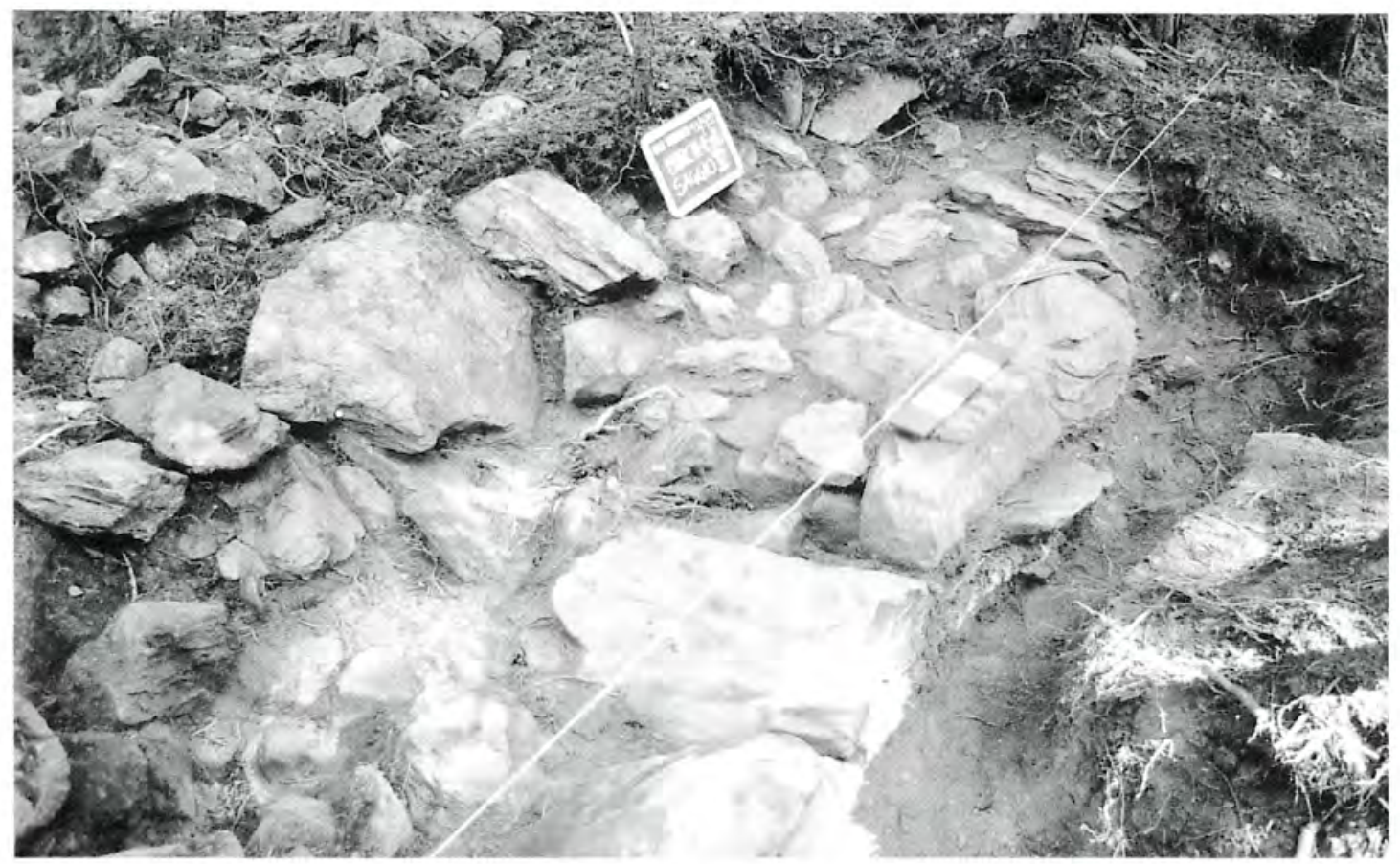

Fig. 16. Birch-Est. Panoramica del saggio V. Si noti la tecnica "a secco" della struttura muraria, con grossi conci laterali, e riempimento centrale costituito da gettate di pietrame di dimensioni minori

Fig. 17. Birch-Est. Panoramica del saggio VI.

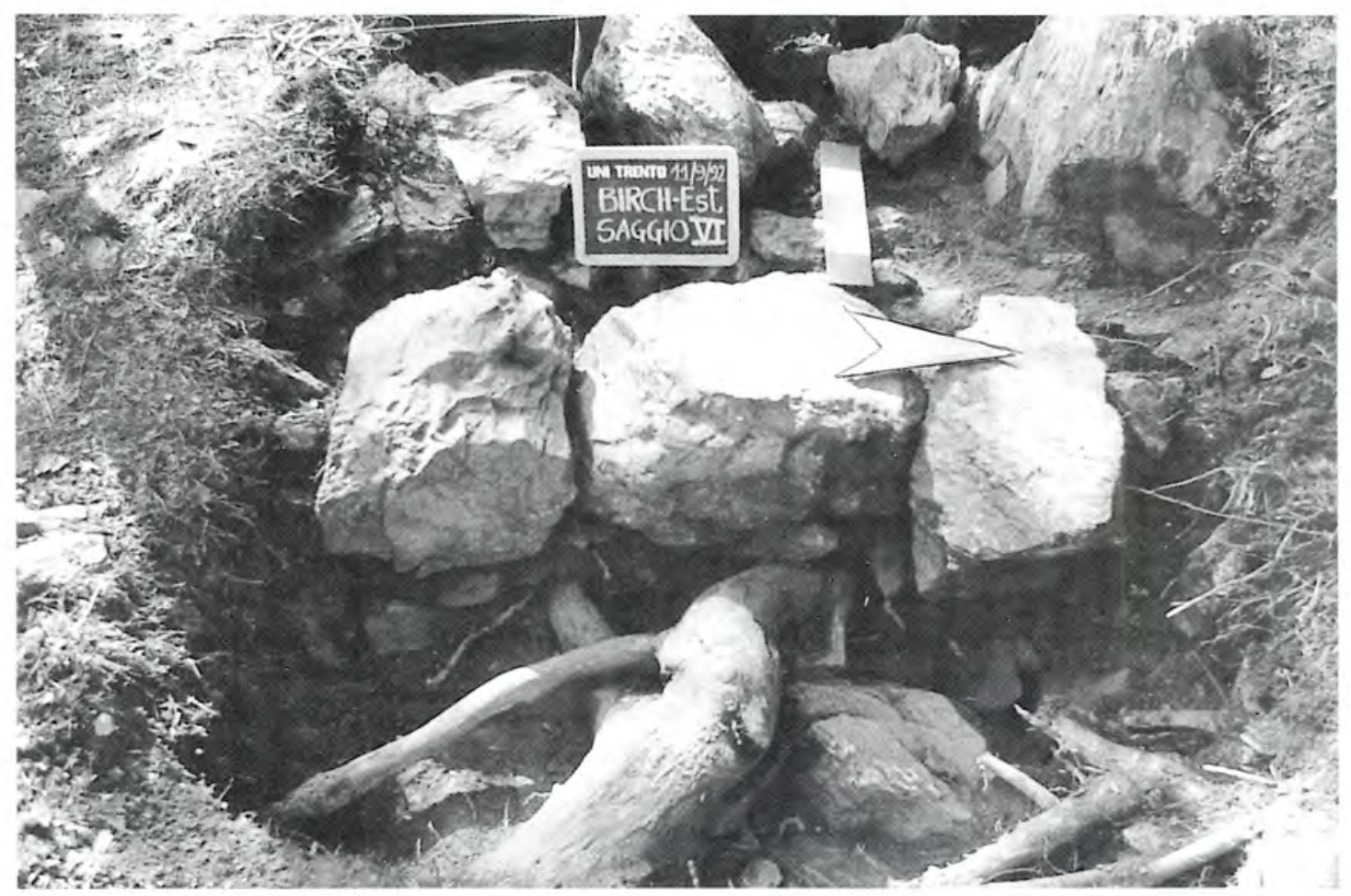



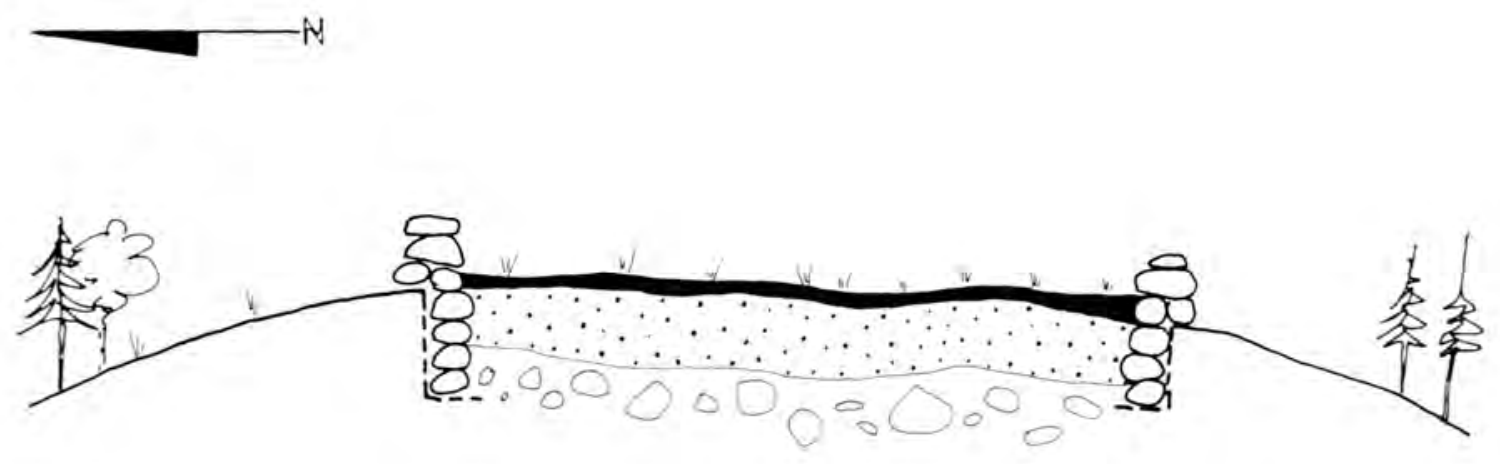

Fig. 18. Birch-Ost. Sezione schematica della sommità del colle. É indicato il probabile taglio in cui è stato alloggiato il muro, forse sfruttando in parte la naturale concavita della cima del colle. Lo strato rappresentato in nero è l'humus boschivo; il deposito reso a puntinato è un fine sedimento limoso a scheletro minuto, che si imposta su un rivestimento di origine morenica. (Dis. dell'A.)

lacunoso proprio sul lato che dà sul maso (Birchhof), ovvero dove era più agevole il reperimento di pietrame e di conci da impiegare eventualmente nella sua costruzione.

Nell' ambito del saggio VI è stato possibile reperire una piccola macina (fig. 20 ) in pietra scistosa presumibilmente preistorica (età del bronzo?) che resta a tutt' oggi il solo elemento utile ad un possibile inquadramento cronologico della frequentazione del sito, se non del manufatto murario descritto: la macina infatti poteva anche giacere nell' ambito di stratificazioni anteriori alla fabbricazione del muro, e non avere pertanto nulla a che fare con esso.

Va osservato che frammenti di macine di porfido dell' età del bronzo sono state rinvenute inserite nel muro di cinta di Sotćiastel (cfr. nota 6 e ralativa bibliografia), che gli scavi hanno rivelato come sicuramente in fase con il relativo abitato fiorito tra il bronzo medio ed un momento iniziale del bronzo recente, Crolli e spoliazioni, ipotizzabili per il muro di Birch-Ost, indiziano forse l' antichità del manufatto. L' assenza di resti archeologici ovvero di stratificazioni relative ad una frequentazione antropica del sito in età preistorica o protostorica nell' area interna al muro si può spiegare o come effetto di lavori di scavo effettuati in rapporto alla vita del Birchhof, per ricavare terreno organico utile alla concimazione dei campi ${ }^{10)}$ ovvero come risvolto di un uso non insediativo

10) A sostegno di questa interpretazione si può forse richiamare la notizia fornita da Innerebner, secondo la quale frammenti ceramici non meglio determinati sarebbero stati scoperti nella selletta che raccorda i due colli Birch (Inner- ebner, 1975: 98). Lavori di spianamento di questo genere, che hanno profondamente modellato la sommità del colle, sono stati ipotizzati anche per Sotćiastel (cfr. nota 6 e relativa bibliografia). 


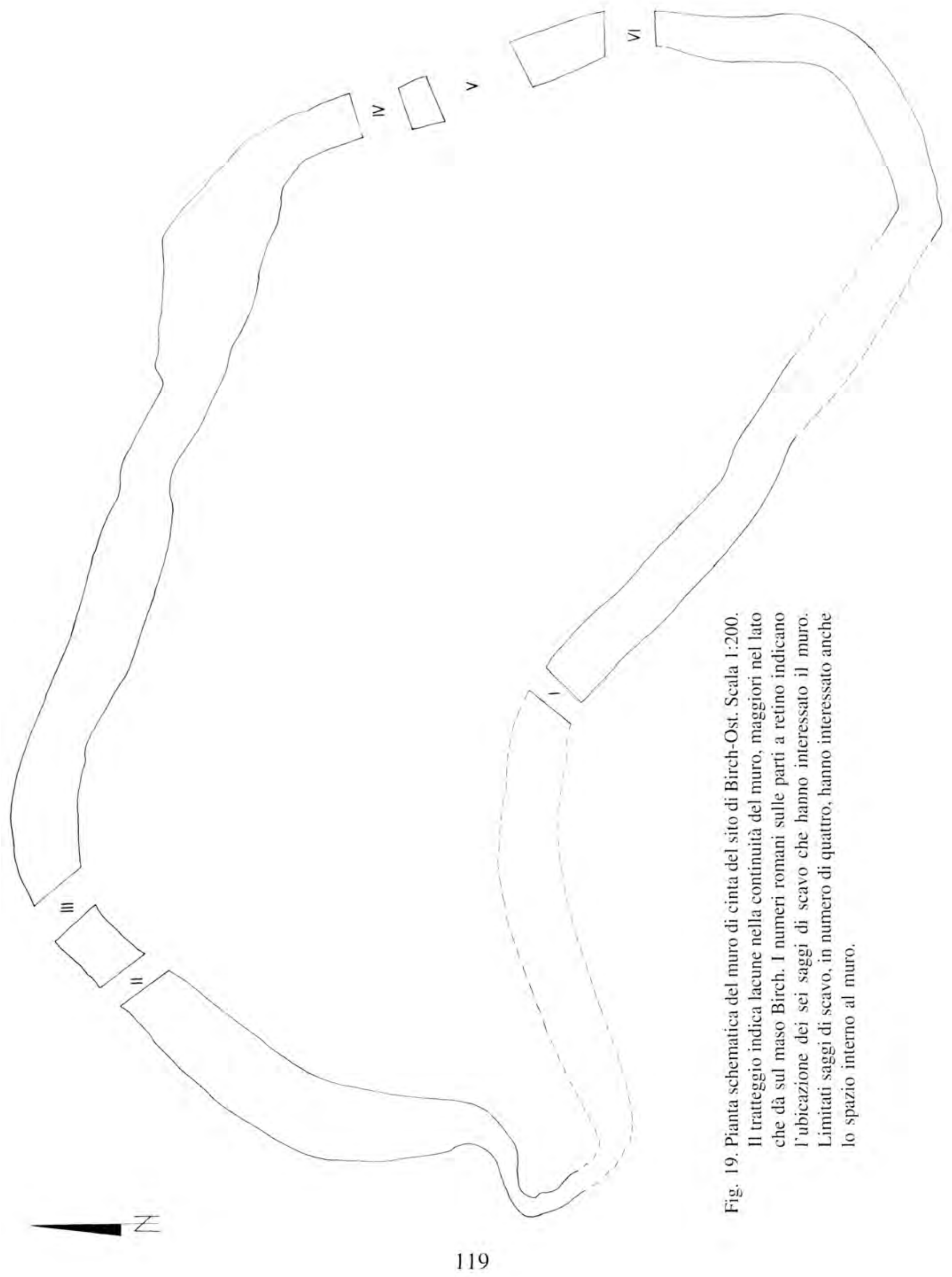




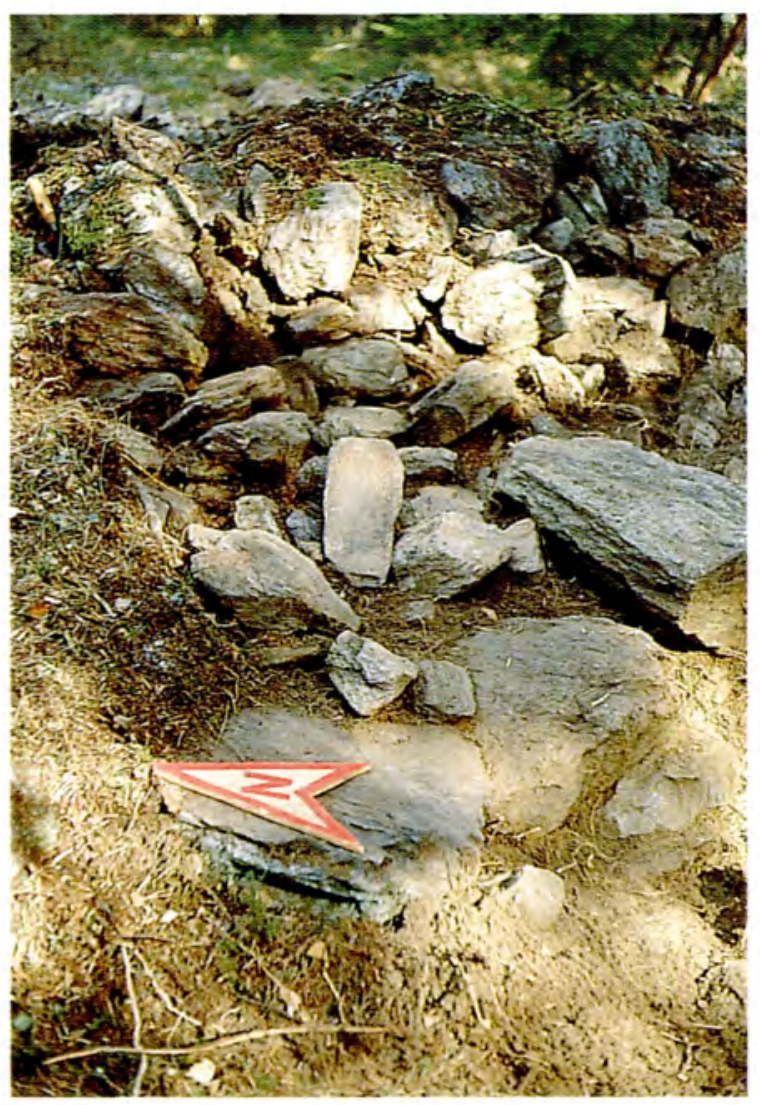

a)

Fig. 20 .

Birch Est:

a) la piccola macina

al momento del rinvenimento

nel corpo del muro (saggio VI);

b) dettaglio della macina.

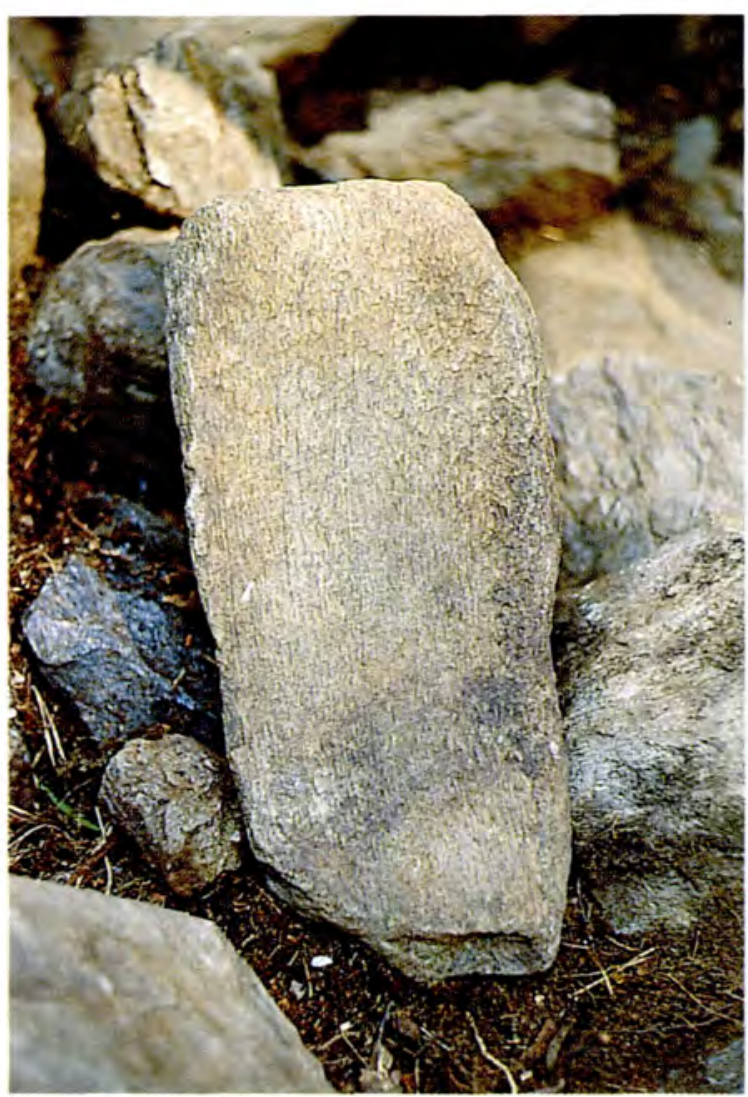


della sommità di Birch-Ost ${ }^{11)}$ che non avrebbe lasciato tracce rilevabili in sede di analisi archeologica.

\section{Ricerche di superficie e sondaggi in Val Gardena.}

\section{1. Col Salames (Wallburg Lasch e dintorni).}

L' attività di ricognizione in Val Gardena ha privilegiato l' analisi di alcune aree campione e in particolare l' esplorazione di alcune emergenze morfologiche non interessate da precedenti ricerche. Tra queste hanno meritato due piccoli saggi il Col Salames, dove sono stati presi in considerazione la Wallburg Lasch, già nota in letteratura (INNEREBNER, 1975), e un riparo sottoroccia, individuato per la prima volta nel corso delle nostre ricerche.

Il Col Salames, in prossimità di Passo Pinëi, comune di Castelrotto, ma geograficamente ancora in Val Gardena, si presenta come una lunga dorsale ad andamento grosso modo est-ovest, caratterizzato da una piccola sommità leggermente sopraelevata, cosparsa di grossi accumuli di pietrame di pezzatura per lo più selezionata. In alcuni punti pare di poter distinguere un vero e proprio allineamento che ripercorre in forma ovaleggiante i limiti dell' emergenza cacuminale.

L' area è risultata interessata dall' intervento di clandestini: un' ampia trincea di forma approssimativamente quadrangolare è stata rinvenuta ricolmata di pietre; nella terra di riporto di tale trincea sono stati recuperati frammenti ceramici probabilmente riferibili all' età del ferro (fig. 21).

Un limitato saggio di scavo di metri quattro di lato ha evidenziato, subito al di sotto della cotica erbosa e a contatto con questa, un coacervo di pietre misuranti mediamente $\mathrm{cm} 40-50$ di asse, prevalentemente di porfido, che riteniamo di poter riferire a strutture antiche sconvolte e crollate ${ }^{12}$.

Tra le pietre al di sotto della cotica erbosa sono venuti alla luce rari frammenti ceramici e numerose ossa combuste. Ad una profondità di trenta centimetri dal suolo attuale è stato possibile recuperare numerosi frammenti di un boccale

11) A sostegno di questa seconda ipotesi depone invece la non eccessiva potenza del muro di cinta, la cui larghezza non sembra superare i $150 \mathrm{~cm}$, utile piuttosto al contenimento di greggi che alla difesa di un presunto abitato; esso avrebbe potuto contare peraltro su uno spazio vitale veramente assai ridotto (meno di 1000 metri quadrati, considerato l' ingombro del muro di cinta), se paragonato per esempio a quello di Sotćiastel (2500 metri quadrati circa secondo le stime di Innerebner, 1975). Per quanto riguarda il rinvenimento di ceramica nella selletta meridionale, al di fuori dello spazio "fortificato", è bene ricordare il caso di Sotćiastel, dove è stato possibile accertare tracce di strutture abitative (buche per palo e resti ceramici sparsi) anche "extra moenia" (cfr. specialmente Dragogna, Walzolgher \& Tecchiati, 1991).

12) Il rimaneggiamento di stratigrafie $e$ strutture deve essere avvenuto principalmente ad opera della fitta vegetazione a conifere e a bosco ceduo, che copre la sommità della collina. 


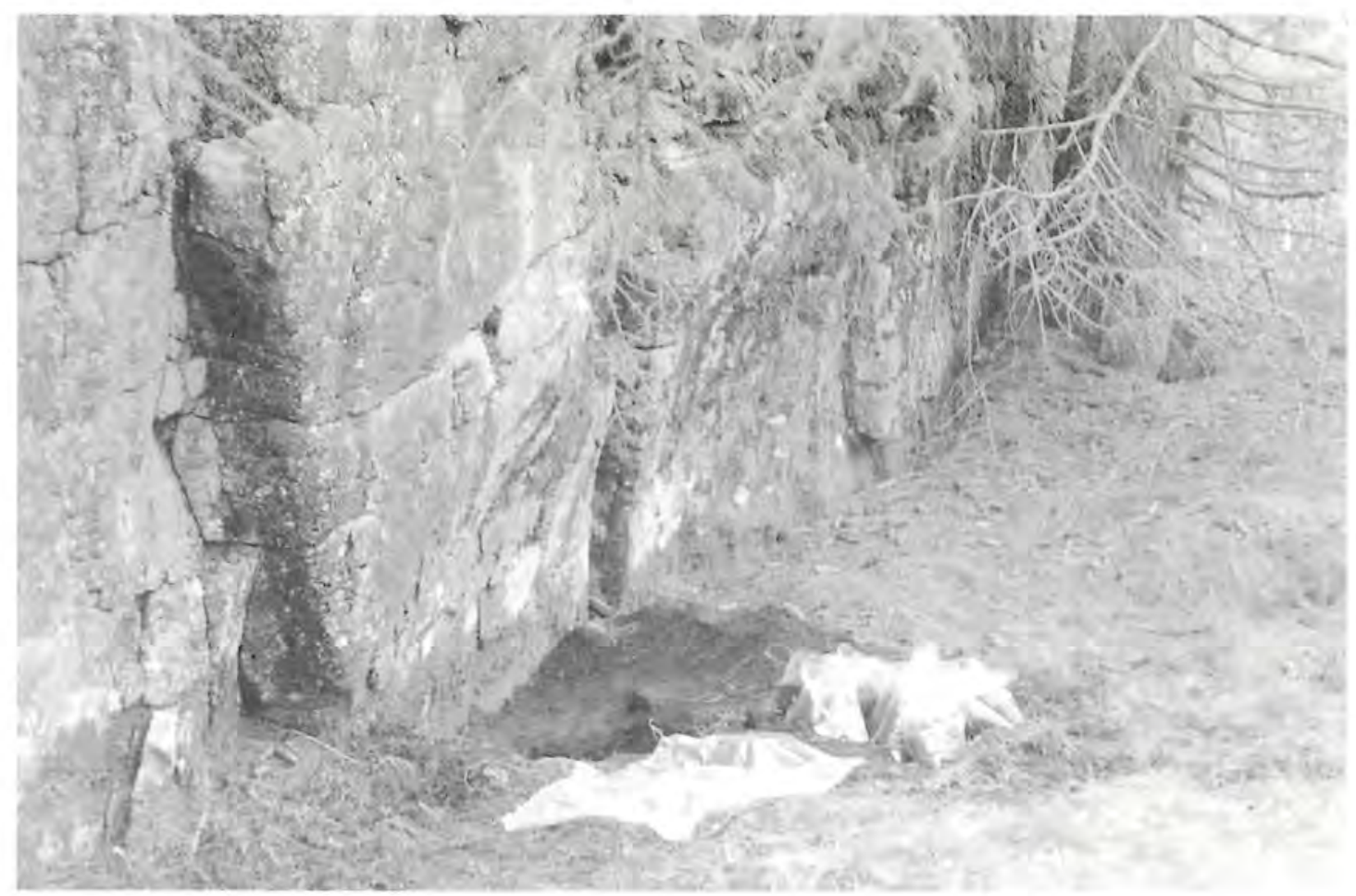

Fig. 21. Passo Pinei. Una lunga parete di roccia porfirica, di altezza modesta, deve aver costituito un riparo occasionale in un momento non meglio precisabile dell 'età del ferro (?). I pochi minuti frammenti ceramici raccolti nel corso di un limitato saggio di scavo ai pedi della paretina, in un terreno intensamente carbonioso, non offrono purtroppo spunti per un più preciso inquadramento cronologico.

di tipo retico (fig. 22). Atteso l' interesse archeologico dell' area il saggio è stato richiuso a questo punto, onde non compromettere con un intervento di troppo limitata ampiezza l' intelligibilità di strutture e stratificazioni evidentemente ancora intatte al di sotto dei "crolli"

Un nuovo sito di sicuro interesse è stato riconosciuto all' estremità occidentale del Col Salames, in vista della Val d' Isarco. Trattasi di un riparo sottoroccia costituito da una lunga parete porfirica leggermente aggettante. Subito al di sotto della cotica erbosa è comparso uno strato fortemente carbonioso, misto a ceneri, che ha restituito pochi frammenti ceramici atipici, forse attribuibili all' età del ferro. Anche in questo caso, esistendo il sospetto che possa trovarsi al di sotto di questo riparo una situazione statigraficamente intatta (forse non limitata alle evidenze protostoriche), meritevole di essere indagata nell' ambito di uno scavo sistematico, il saggio si è interrotto a trenta centimetri dal piano di calpestio attuale e la piccola trincea è stata ricoperta ${ }^{14)}$.

13) A seguito del nostro intervento il sito è stato compreso nelle aree soggette a vincolo ambientale e paesaggistico della Provincia Autonoma di Bolzano.
14) Istanze di tutela ci inducono a non specificare ulteriormente la precisa localizzazione del riparo. 

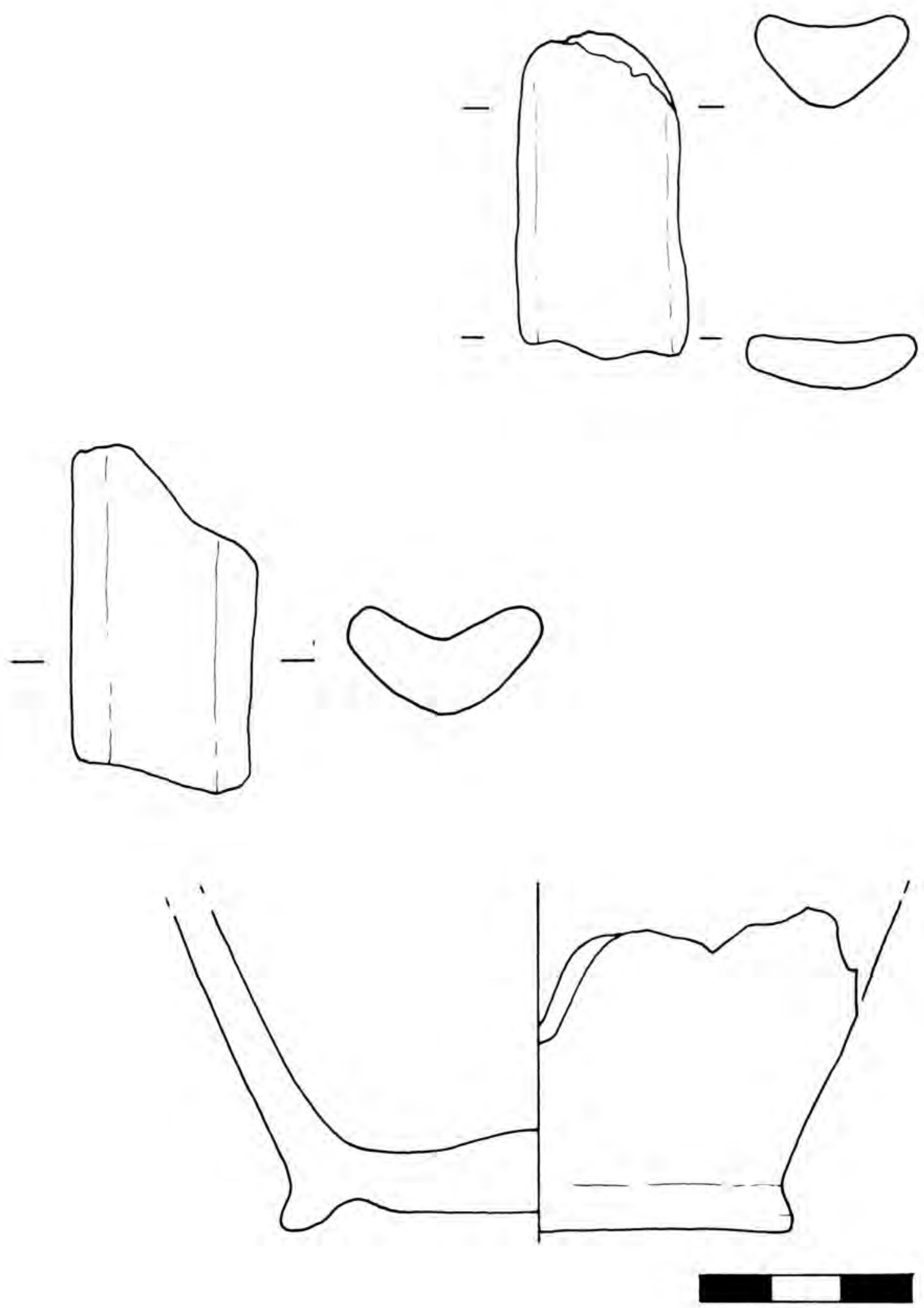

Fig. 22. Wallburg-Lasch. Frammenti ceramici pertinenti probabilmente ad un medesimo boccale retico frantumato al suolo. 


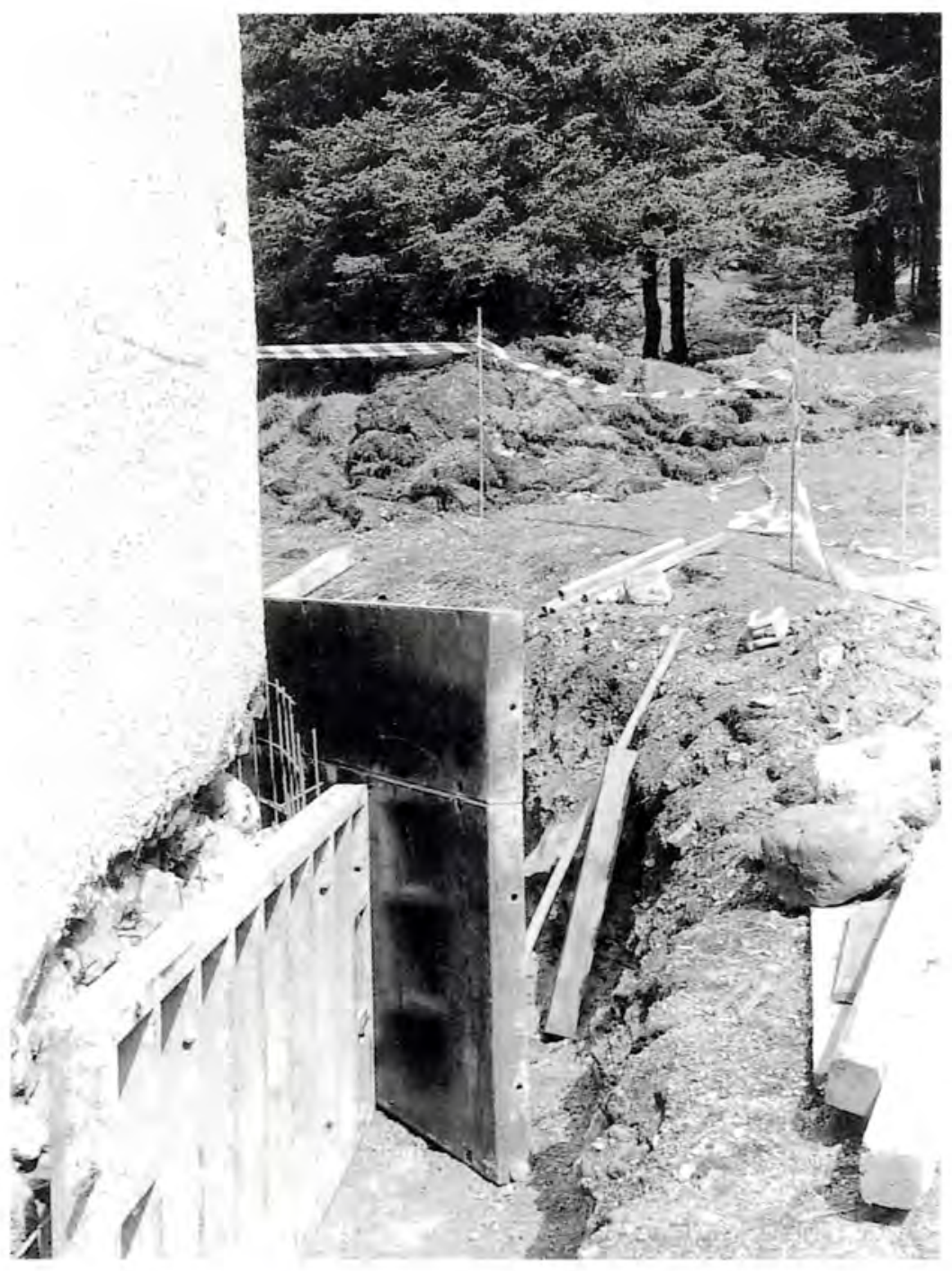

Fig. 23.

Cappella Vallunga. Dettaglio dell'edificio durante $i$ lavori di ristrutturazione (drenaggio).

\section{2. Selva di Val Gardena - località "Ciastelat".}

Il "Ciastelat" è un colle eminente che si affaccia in posizione panoramica eccezionalmente favorevole sul "Plan" e sul corso del torrente Gardena. La sommità si presenta come una sottile lingua rocciosa fittamente ricoperta dalla vegetazione sempreverde a conifere. Presentando lati estremamente scoscesi bisogna supporre per questo sperone di roccia una ottima difendibilità. In assenza di vegetazione, la visuale potrebbe spaziare libera a 365 gradi su un' ampia porzione della Val Gardena e sulla laterale Vallunga.

Lungo il sentiero che porta sulla sommità della collina sono stati notati numerosi punti in cui il terreno, in corrispondenza di tagli effettuati nel pendio per il tracciato del sentiero stesso, è particolarmente organico e carbonioso. Minuti resti faunistici si rinvengono abbastanza frequentemente, purtroppo non associati ad altri indicatori archeologici utili ad un loro inquadramento cronologico. 


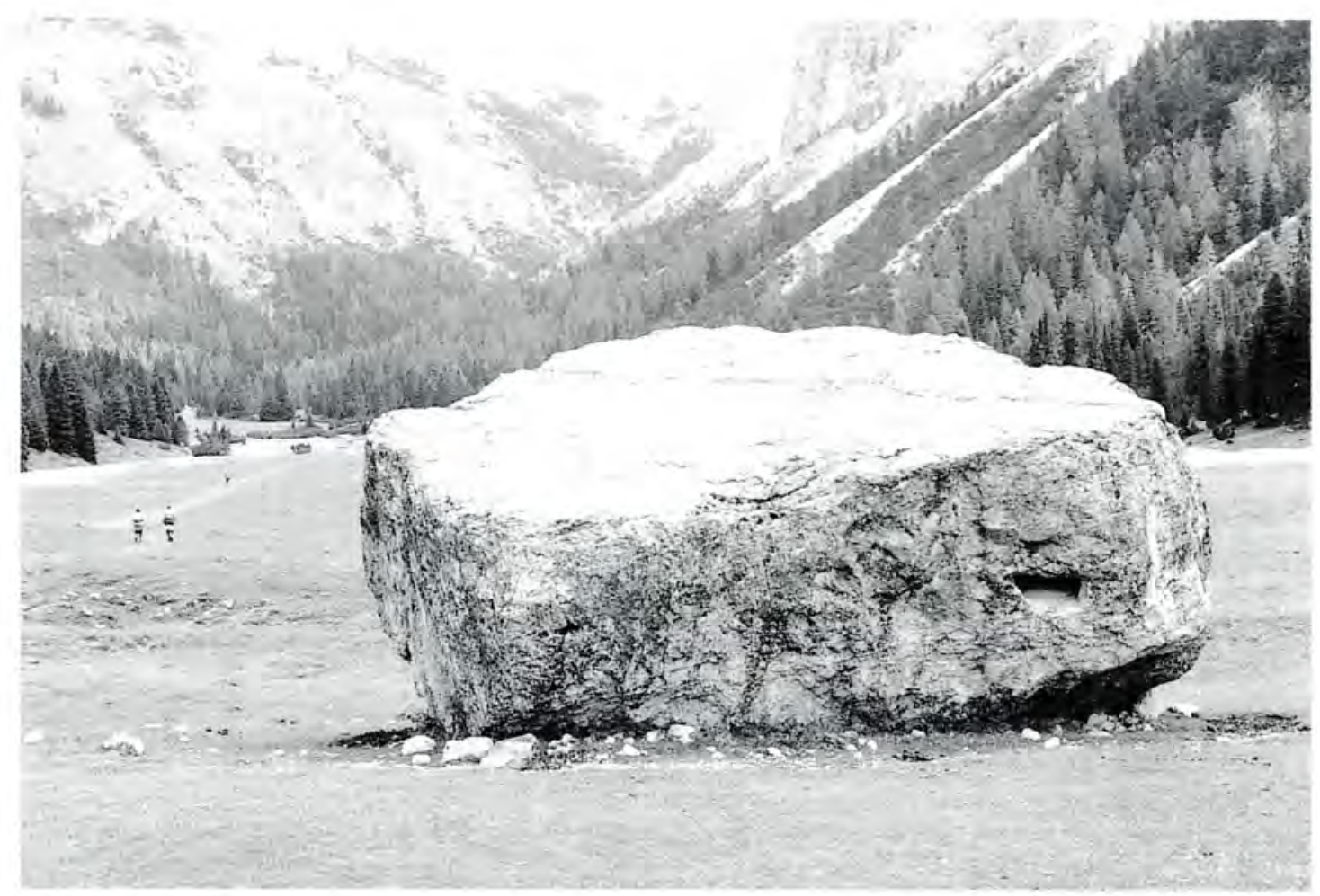

Fig. 24. Riparo Vallunga. Il masso erratico, di dimensioni veramente ridotte (h. massima $m 3$, lunghezza $m$ 6, larghezza $m 4$ circa), offriva, ai cacciatori di un momento non meglio precisabile del mesolitico, un modesto riparo, sfruttato talvolta ancora oggi dai numerosi escursionisti che frequentano la valle.

Pochi metri al di sotto della cima, in un punto in cui è stata collocata una panchina in posizione panoramica, è stata osservata, nell' ambito di una sezione occasionale del pendio, una quantità di pietre forse in origine legate in calce ${ }^{15}$. $\mathrm{Al}$ di sotto di tale coacervo di pietre sono state osservate altre pietre, almeno in parte diverse per pezzatura, colore, dimensioni, litotipo, pertinenti forse ad una "struttura" precedente. La pulitura della sezione occasionale di cui sopra non ha apportato alcun dato archeologico, così come alcuni limitati saggi di scavo aperti in diversi punti della sommità della collina sul lato che dà sul "Plan".

Nonostante la sostanziale impossibilità, allo stato attuale delle ricerche, di attribuire al Ciastelat un preciso significato archeologico, si deve riconoscere che la favorevole posizione geografica e strategica, e il toponimo stesso, non infrequentemente considerato indicatore di siti pre- o protostorici d' altura, propongono questa emergenza morfologica come tra le più interessanti e promettenti dell' intera Val Gardena, e senz' altro tra le più meritevoli di attenzione da parte della ricerca archeologica da campo.

15) "Sfatticcio" di calce è presente nei vuoti tra le pietre, interpretabili forse, preliminarmente, come crollo di una struttura in muratura. 


\section{3. Val/Vallunga}

Il survey in Vallunga ha riguardato in pratica l' intera estensione della valle che si stacca verso settentrione a monte dell' abitato di Selva di Val Gardena. Due siti in particolare sono stati presi in considerazione: Cappella Vallunga e un masso erratico in località Pra da Ri.

Notizie in possesso del Signor Herwig Prinoth volevano che durante gli scassi effettuati per il drenaggio perimetrale della cappella che sorge al principio del sentiero turistico della Vallunga fosse venuto alla luce uno strato carbonioso sepolto a media profondità in una situazione di leggero pendio (fig. 23).

In effetti, a $140 \mathrm{~cm}$ circa di profondità dal suolo attuale, abbiamo notato uno strato fortemente organico, nerastro, untuoso al tatto, coperto da un sedimento a marcata componente limo-argillosa, chiaro, privo di scheletro. $\mathrm{Si}$ impostava a sua volta su un suolo argilloso, a quanto pare varvato, che presentava un colore bruno-grigiastro. Nessuna evidenza archeologica particolare è stata rilevata nel-l'ambito di questa sezione. Pare infatti di poter affermare che tale strato, della potenza complessiva di $\mathrm{cm} 5$ circa, sia da riconnettere all' esistenza in loco, in un periodo sconosciuto ${ }^{16}$, di un bacino idrico. Lo stesso strato organico si configura forse come l' effetto della decomposizione di resti vegetali in un ambiente asfittico.

Nelle zolle accantonate dall' escavatrice subito a monte della cappella si è creduto di ravvisare, in un minuscolo frammento di consistenza vetrosa, trasparente, fratturato in maniera concoide, un frammento di cristallo di rocca ${ }^{17}$. Minuscoli frammenti di selce contenuti parimenti nella zolla, appaiono di difficile interpretazione a causa della loro atipicità.

In località Pra da Ri, ad un' ora di cammino da Cappella Vallunga, e in prossimità del sentiero turistico, si trova un masso erratico calcareo, con almeno una parete bene aggettante (fig. 24). In corrispondenza di tale aggetto, in un punto in cui il manto vegetale si presentava strappato, in un terreno molto carbonioso, sono state rinvenute numerose minutissime schegge di selce di colore rosso mattone e grigio. Tra queste nessuna può essere considerata prodotto di litotecnica, anche se l' eterogeneità dei tipi cromatici permette di avanzare preliminarmente l' ipotesi di una loro origine antropica. Le dimensioni millimetriche delle schegge di selce, e la quota del riparo (m 2000 circa slm) permettono di porre una eventuale frequentazione antropica del sito nell'ambito del mesolitico.

16) Il periodo di attività di tale bacino idrico dovrebbe comunque essere anteriore alla costruzione della cappella, dal momento che i sedimenti da esso depositati appaiono tagliati dalle fosse di fondazione dei muri dell' edificio.
17) Esiste a mio avviso la possibilità che possa trattarsi di un frammento di vetro. Va osservato però che la ricerca di superficie non ha evidenziato altri resti archeologici di età recente $\mathrm{o}$ subrecente. 


\section{Bibliografia}

Bagolini, B. / Tasca, G. / Tecchiati, U. (1989): "Relazione preliminare e risultati della prima campagna di scavi nell' insediamento dell' età del bronzo di Sotćiastel". In: Ladinia XIII. 5-33.

Bagolini, B. / Tasca, G. / Tecchiati, U. (1990-91): 'L' abitato di Sotćiastel (S. Leonardo in Badia, Bolzano)". In: Rassegna di archeologia 10. $630-631$

Bagolini, B. / Tecchiati, U. (1993a: in corso di stampa): “Osservazioni sul popolamento delle valli ladine tra Neolitico ed età del bronzo nel quadro della preistoria del bacino atesino". In: AAVV: "Archeologia nelle Dolomiti. Ricerche e ritrovamenti nelle Valli del Sella dall' età della pietra alla romanità", Istitut Cultural Ladin "Majon di Fashegn" e Istitut Cultural Ladin "Micurà de Rü".

Bagolini, B. / Tecchiati, U. (1993b; in corso di stampa): "Sotćiastel: un abitato fortificato della media e recente età del bronzo in Val Badia (Comune di San Leonardo)". In: AAVV: Archeologia nelle Dolomiti. Ricerche e ritrovamenti nelle Valli del Sella dall' età della pietra alla romanità. Istitut Cultural Ladin "Majon di Fashegn" e Istitut Cultural Ladin "Micurà de Rü".

Bombonato, G. (1993; in corso di stampa): "Un' indagine archeologica in Val di Fassa". In: AAVV: Archeologia nelle Dolomiti. Ricerche e ritrovamenti nelle Valli del Sella dall' età della pietra alla romanità. Istitut Cultural Ladin "Majon di Fashegn" e Istitut Cultural Ladin "Micurá de Rü”, nel 1993.

Dragogna, G. / Tecchiati, U. / Walzolgher, E. (1991): "La terza campagna di scavi nell' abitato dell' età del bronzo di Sotćiastel in Val Badia". In: Ladinia XV, 5-45.

Innerebner, G., (1961): "Südtiroler Wallburgenstatistik. Gruppe IX: Pustertal und Enneberg". In: Der Schlern 35. 134-145.

Innerebner, G. (1975): Die Wallburgen Südtirols. Bd. 1: Pustertal, Bozen.

Lunz, R. (1979): "Zur Vor- und Frühgeschichte von Abtei und Enneberg mit Ausblicken auf Gröden”. In: Ladinia III, 147-163.

Prinoth. R. (1988): Urgeschichte des Grödnertales. Dissertation zur Erlangung des Doktorgrades an der Geisteswissenschaftlichen Fakultät der Leopold-Franzens Universität Innsbruck.

Richter-Santifaller, B. (1937): Die Ortsnamen von Ladinien. Innsbruck.

Tecchiati, U. (1990): “Aggiornamento sullo stato delle ricerche archeologiche nell' abitato dell' età del bronzo di Sotćiastel in Val Badia (Bolzano)". In: Ladinia XIV, 21-56.

Zago, Z. R. (1993; in corso di stampa): "Ricerca archeologica con il metodo geoelettrico o della resistività in Val di Fassa". In: AAVV, Archeologia nelle Dolomiti. Ricerche e ritrovamenti nelle Valli del Sella dall' età della pietra alla romanità. Istitut Cultural Ladin "Majon di Fashegn" e Istitut Cultural Ladin "Micurá de Rü". 